Kybernetes: The Intern. Journ. of Systems \& Cybernetics 32, No. 7/8, pp. 976-1004 (2003)

(a special issue on new theories about time and space, Eds.: L. Feng, B. P. Gibson and Yi Lin)

\title{
Scanning the structure of ill-known spaces: Part 2. Principles of construction of physical space
}

\author{
Michel Bounias $^{(1)}$ and Volodymyr Krasnoholovets ${ }^{(2)}$ \\ (1) BioMathematics Unit(University/INRA, France, and IHS, New York, USA), \\ Domain of Sagne-Soulier, 07470 Le Lac d'Issarles, France \\ $\left(^{2}\right)$ Institute of Physics, National Academy of Sciences, \\ Prospect Nauky 46, UA-03028, Kyïv, Ukraine
}

\begin{abstract}
An abstract lattice of empty set cells is shown to be able to account for a primary substrate in a physical space. Space-time is represented by ordered sequences of topologically closed Poincaré sections of this primary space. These mappings are constrained to provide homeomorphic structures serving as frames of reference in order to account for the successive positions of any objects present in the system. Mappings from one to the next section involve morphisms of the general structures, standing for a continuous reference frame, and morphisms of objects present in the various parts of this structure. The combination of these morphisms provides space-time with the features of a nonlinear generalized convolution. Discrete properties of the lattice allow the prediction of scales at which microscopic to cosmic structures should occur. Deformations of primary cells by exchange of empty set cells allow a cell to be mapped into an image cell in the next section as far as mapped cells remain homeomorphic. However, if a deformation involves a fractal transformation to objects, there occurs a change in the dimension of the cell and the homeomorphism is not conserved. Then the fractal kernel stands for a "particle" and the reduction of its volume (together with an increase of its area up to infinity) is compensated by morphic changes of a finite number of surrounding cells. Quanta of distances and quanta of fractality are demonstrated. The interaction of a moving particle-like deformation with the surrounding lattice involves a fractal decomposition process that supports the existence and properties of previously postulated inerton clouds as associated to particles. Experimental evidence of the existence of inertons are reviewed and further possibilities of experimental proofs are proposed.
\end{abstract}

Key words: origin of particles; origin of quantum property; origin of gravitation; cosmic features from microscale properties; nothingness singleton.

PACS classification: $02.10 . \mathrm{Cz}$; - set theory; 02.40.Pc - general topology; 03.65.Bz - foundations, theory of measurement, miscellaneous theories. 


\section{Introduction}

Former parts of this study were dealing with some founding principles about how to assess in the more accurate though general way possible how one can define the space of magmas (that is, the sets, combination rules and structures) in which a given proposition can be demonstrably shown to be valid (Bounias, 2001; Bounias and Krasnoholovets, 2003). Such a space, when identified, is called a probationary space (Bounias, 1997; 2001). Here, it will be presented the formalism which leads from existence of abstract (e.g. purely mathematical) spaces to the justification of a distinction between parts of a physical space that can be said empty and parts which can be considered as filled with particles. This question is thus dealing with a possible origin of matter and its distribution, and changes in this distribution gives raise with motion, that is with physics, in the sense pointed by de Broglie and by Dirac (see Rothwarf, 1998). Experimental evidence and propositions for further verifications will then be presented and discussed.

Recent findings (Krasnoholovets and Ivanovsky, 1993; Krasnoholovets 1997) in the realm of fundamental physics supports the prediction that an abstract lattice whose existence originates in the existence of the empty set, is able to correctly account for various properties of our observed space-time at both microscopic and cosmic scales. The model of Krasnoholovets and Byckov (2000), Krasnoholovets (2000), proposed a new research methodology based on some practical standpoints. Specifically (see e.g. Okun, 1988), the values of the constants of electromagnetic, weak and strong interactions as functions of distances between interacting particles converge to the same at a scale about $10^{-28} \mathrm{~cm}$. This suggests that a violation of space homogeneity took place at this size. The model proceeds from the assumption that all quantum theories (quantum mechanics, electrodynamics, chromodynamics and others) are in fact only phenomenological: accordingly, for the understanding of real processes occurring in the real microworld, one needs a submicroscopic approach which, in turn, should be available for all peculiarities of the microstructures of real space. In other terms, gauges for the analysis of all components of the observable universe should belong to an ultrafilter, as argued in Part 1 of this study. The investigations about the model of inertons (Krasnoholovets, 1997, 2000; 2001a,b,c) has suggested that a founding cellular structure of space shares discrete and continuous properties, which is also shown to be consistent with the abstract theory of foundations of existence of a physical space (Bounias and Bonaly, 1997).

\section{Preliminaries}

\subsection{About gaps in former assessment of probationary spaces}

2.1.1. Quantum mechanics. Quantum mechanicsis founded on the calculation of the probability that a particle is present in a given volume of space. This theoretical approach postulates the existence of undefined objects called corpuscles, and does not state about the structure and properties of any embedding medium, which is even considered as forbidden (Blokhintsev, 1981). Only recently, however, was raised the need that this medium, sometimes called the void, should be a space allowing the formation of pairs of particles and antiparticles (see Boyer, 2000, for review), so as 
to justify for the existence of a material world. However, this postulate just displace the question of the corresponding embedding medium, which is supposed not to exist independently from the photons but is often considered as if it was independent from at least large matter masses. Parameter time is not basically but implicitly present in the foundations of quantum physics. The concept of velocity of wave propagation and its expressions in the uncertainty principle, the Bell's inequalities, etc. emerge from further developments of the theory.

2.1.2. Relativity. The relativistic theory postulates the existence of frames of references and of the validity of some particular cases of measure, used as classical metrics, still without consideration for the embedding medium (Blokhintsev, 1976). It also postulates the primary existence of parameter time and the consistency of the possibility of motion in an undefined space, sometimes identified with "void", with the properties of this "void". However, the limitation found for the velocity of light up to the cosmological constant through electromagnetism, and the proposition of curvature of space implicitly impose some conditions on some relevant embedding medium (Einstein, 1920; Marinov, 1996; Keilman, 1998, and many others).

\subsection{Assessment of existence of a space-time-like structure}

A former conjecture (Bonaly, 1992) stated that a characteristic of a physical space is that it should be in some way observable. This implies that an object called the "observer" should be able to interact with other objects said "observed". In order to make no confusion with the usual vocabulary of systems theory, we will instead refer to the "perceiver" and the "perceived" objects. The conjecture implied that perceived objects should be topologically closed, otherwise they would offer no frontier to allow a probe to reflect their shape. Therefore, the first step of the work was to assess the existence of closed topological structures, and a proof was given that the intersection of two spaces having nonequal dimensions owns its accumulation points and is therefore closed. We propose here a shorter alternative proof.

Theorem 2.2. The intersection of two connected spaces with nonequal dimension is topologically closed.

Alternative proof. Let $\mathrm{E}^{\mathrm{n}}$ and $\mathrm{E}^{\mathrm{m}}$ be two spaces with topological dimensions $\mathrm{n}, \mathrm{m}$

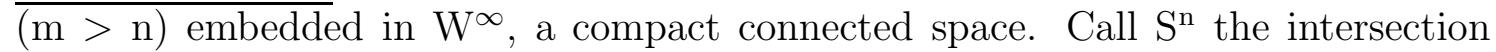
$\mathrm{E}^{\mathrm{n}} \cap \mathrm{E}^{\mathrm{m}}$ and call $\mathrm{X}^{\mathrm{n}}$ the complementary of $\mathrm{S}$ in $\mathrm{E}^{\mathrm{n}}$. Consider the continuity of mappings in $\mathrm{W}^{\infty}$ inducing continuity to $\mathrm{E}^{\mathrm{n}}$ and $\mathrm{E}^{\mathrm{m}}$ : the neighborhood of any point in $\mathrm{E}^{\mathrm{m}}$ is the mapping of a neighborhood of a point in $\mathrm{X}^{\mathrm{n}}$. Suppose $\mathrm{S}^{\mathrm{n}}$ is open: then, since the union of open sets is open, then the entire $\mathrm{E}^{\mathrm{m}}$ is neighborhood of any point in $\mathrm{S}^{\mathrm{n}}$. Thus, there would exist a bijective mapping of opens of $\mathrm{X}^{\mathrm{m}}$ on opens of $\mathrm{S}^{\mathrm{n}}$. In particular, a open subset of $(\mathrm{n}+2)$ points in $\mathrm{S}^{\mathrm{n}}$ could be homeomorphic to a $(\mathrm{n}+\mathrm{l})$-simplex in $\mathrm{E}^{\mathrm{m}}$. This is impossible because two spaces with nonequal dimensions cannot be homeomorphic. Therefore, $\mathrm{S}^{\mathrm{n}}$ is closed.

The closed 3-D intersections of parts of a n-space (with $n \leq 3$ ) own the properties of Poincaré sections (Bonaly and Bounias, 1995). Then, given a manifold of such sections, the mappings of one into another section provide an ordered sequence of corresponding spaces in which closed topological structures are to be found: this 
accounts for a time-like arrow. Since the Jordan-Veblen theorem states that any path connecting the interior of a closed to an outside point has a nonempty intersection with the frontier of the closed, interactions between closed objects are allowed: this accounts for physical interactions. Furthermore, if such a path is connected to a converging sequence of mappings, the fixed points (of Banach type, here) will stand for perceptions of the outside. Moreover, since the Brouwer's theorem states that in a closed, all continuous mappings have a fixed point, and that the brain represents a compact complete space in which mappings from a topological into a discrete space are continuous, there exists an associate set of fixed points (of the Brouwer's type) standing for the self (Bounias and Bonaly, 1997). Finally, spaces of topologically closed parts account for interaction and for perception, thus they meet the properties of physical spaces.

In a former conjecture, Bonaly and Bounias (1993) proposed that the fundamental metrics of our space-time should be represented by a convolution product where the embedding part U4 would be described by the following relation:

$$
\mathrm{U} 4=\int\left(\int_{\mathrm{dS}}(\mathrm{d} \overrightarrow{\mathrm{x}} \cdot \mathrm{d} \overrightarrow{\mathrm{y}} \cdot \mathrm{d} \overrightarrow{\mathrm{z}}) * \mathrm{~d} \Psi(\mathrm{w})\right)
$$

where $d S$ is an element of space-time, and $d \Psi(w)$ a function accounting for the extension of $3-\mathrm{D}$ coordinates to the 4 th dimension through convolution $(*)$ with the volume of space. Formal proofs of this structure will be provided below.

\section{On foundations of space-time}

\subsection{Space-time as a topologically discrete structure}

How two Poincaré sections are mapped is assessed by using a natural metrics of topological spaces: the set-distance, first established for two sets (Bounias and Bonaly, 1996) and further generalized to manifolds of sets (Bounias, 1997), Figure 1. In brief: let $\Delta(\mathrm{A}, \mathrm{B}, \mathrm{C}, \ldots)$ the generalized set distance as the extended symmetric difference of a family of closed spaces:

$$
\Delta\left(\mathrm{A}_{\mathrm{i}}\right)_{\mathrm{i} \in \mathrm{N}}=\complement_{\cup\{\mathrm{Ai}\}} \cup_{i \neq j}\left(\mathrm{~A}_{\mathrm{i}} \cap \mathrm{A}_{\mathrm{j}}\right) .
$$

The complementary of $\Delta$, that is $\cup_{i \neq j}\left(A_{i} \cap A_{j}\right)$ in a closed space is closed. It is also closed even if it involves open components with nonequal dimensions. Thus, in this system, $\mathfrak{m}\left\langle\left\{A_{i}\right\}\right\rangle=\cup_{i \neq j}\left(A_{i} \cap A_{j}\right)$ has been called the "instant", that is the state of objects in a timeless Poincaré section (Bounias, 1997). Since distances $\Delta$ are the complementaries of objects, the system stands as a manifold of open and closed subparts. Mappings of these manifolds from one into another section which preserve the topology stand for a reference frame in which the "analysis situ" (the original name for "topology") will allow to characterize the eventual changes in the configuration of some components: if morphisms are observed, then this will be interpretable as a motion-like phenomenon when comparing the state of a section to the state of the mapped section.

It should be noted that the spaces referred above can exist upon acceptance of the existence of the empty set as a primary axiom (Bounias and Bonaly, 1997), with consequences which will be addressed below. 


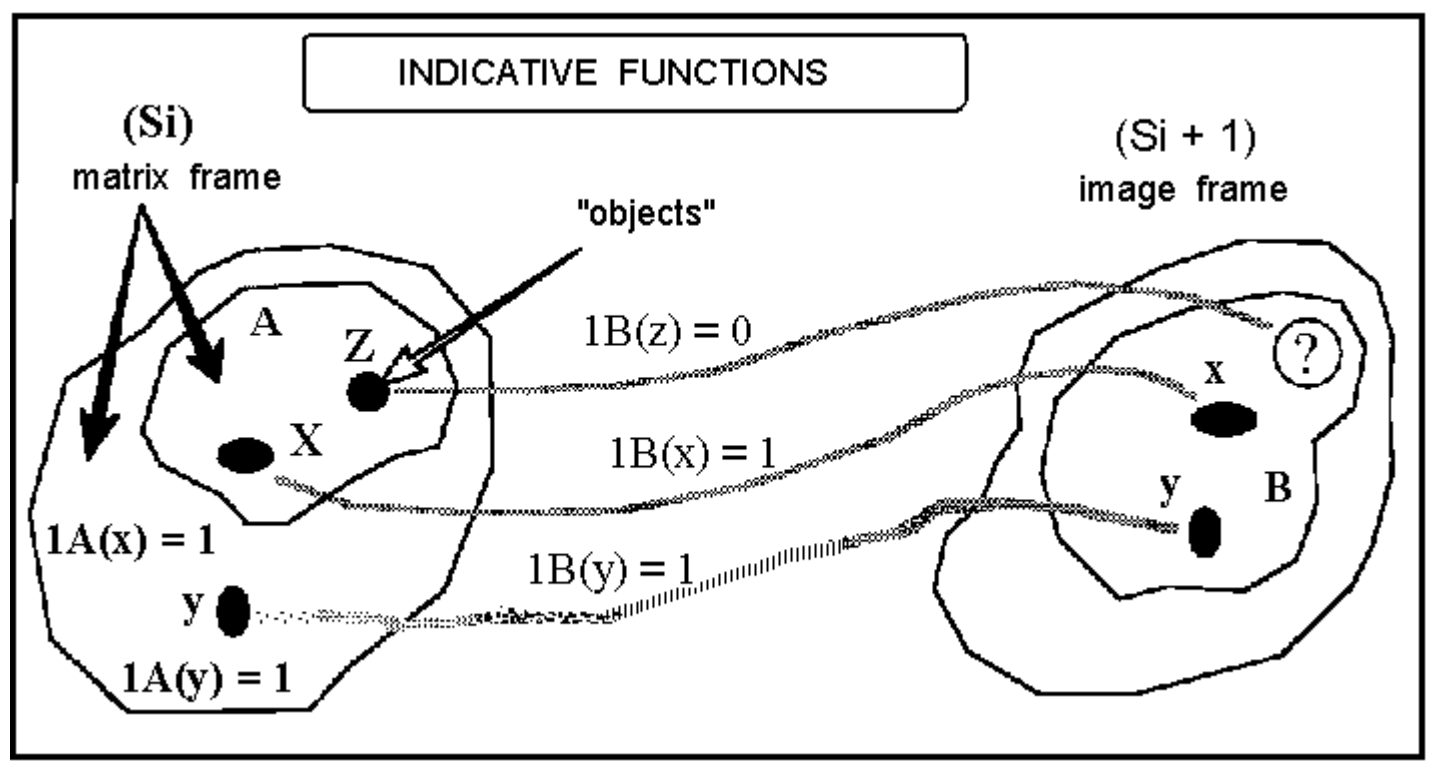

Figure 1: The mappings of a Poincaré section $\mathrm{S}(\mathrm{i})$ into a section $\mathrm{S}(\mathrm{i}+1)$ imposes the conservation of the topologies of the general structure of the mapped spaces, so that changes in the position of objects situated inside these structures can be characterized. A closed should be mapped into an equivalent closed, an open into an equivalent open. The situation of points $(\mathrm{x}, \mathrm{y}, \ldots)$ with respect to these reference structures $(\mathrm{A}, \mathrm{B}, \ldots)$ are described by indicatrix functions $1 \mathrm{~A}(\mathrm{x}), 1 \mathrm{~B}(\mathrm{x}), \ldots$

Lemma 3.1. The set-distance provides a set with the finer topology and the setdistance of nonidentical parts provides a set with an ultrafilter.

Proof. The set-distance is founded on $\{\cap \cup \in\}$ and it suffices to define a topology since union and intersection of set-distances are distances, including $\Delta(\mathrm{A}, \mathrm{A})=\varnothing$. The latter case must be excluded from a filter, which is nonempty. Then, since any filter and any topology is founded on $\{\cap \cup \in \notin \supset\}$, it is provided with $\Delta$. Conversely, regarding a topology or a filter founded on any additional property $(\perp)$, this property is not necessarily provided to a $\Delta$-filter. The topology and filter induced by $\Delta$ are thus respectively the finer topology and an ultrafilter.

The mappings of both distances and instances from one to another section can be described by a function called the "moment of junction", since it has the global structure of a momentum (Figure 2). Consider the particular case of the homeomorphic sequence of mappings of the general topology of the system: this provides a kind of reference frame, in which it will become possible to assess the changes in the situation of points and sets of points eventually present within these structures. The origin of such points will be addressed in the next section. Here, just consider point $(\mathrm{x}, \mathrm{y}, \mathrm{z}, \ldots)$ belonging to either of closed and open parts. For any $\mathrm{x}$ belonging to a set $\mathrm{Ei}$ in a section $\mathrm{S}(\mathrm{i})$, an indicatrix function $1(\mathrm{x})$ is defined by the correspondence of $\mathrm{x}$ with some $\mathrm{c}(\mathrm{x})$ in $\mathrm{S}(\mathrm{i}+1)$ :

$$
\begin{aligned}
& \mathrm{x} \in \mathrm{E}(\mathrm{i}), 1_{\mathrm{Ei}}(\mathrm{x})=1 \text { iff } \mathrm{x} \in \mathrm{Ei} ; 1_{\mathrm{Ei}}(\mathrm{x})=0 \text { iff } \mathrm{x} \notin \mathrm{Ei} \\
& \mathrm{c}(\mathrm{x}) \in \mathrm{E}(\mathrm{i}+1), 1_{(\mathrm{Ei}+1)}(\mathrm{x})=1 \text { iff } \mathrm{c}(\mathrm{x}) \in \mathrm{E}(\mathrm{i}+\mathrm{l}) \\
& 1_{(\mathrm{Ei}+1)}(\mathrm{x})=0 \text { iff } \mathrm{c}(\mathrm{x}) \notin \mathrm{E}(\mathrm{i}+1)
\end{aligned}
$$




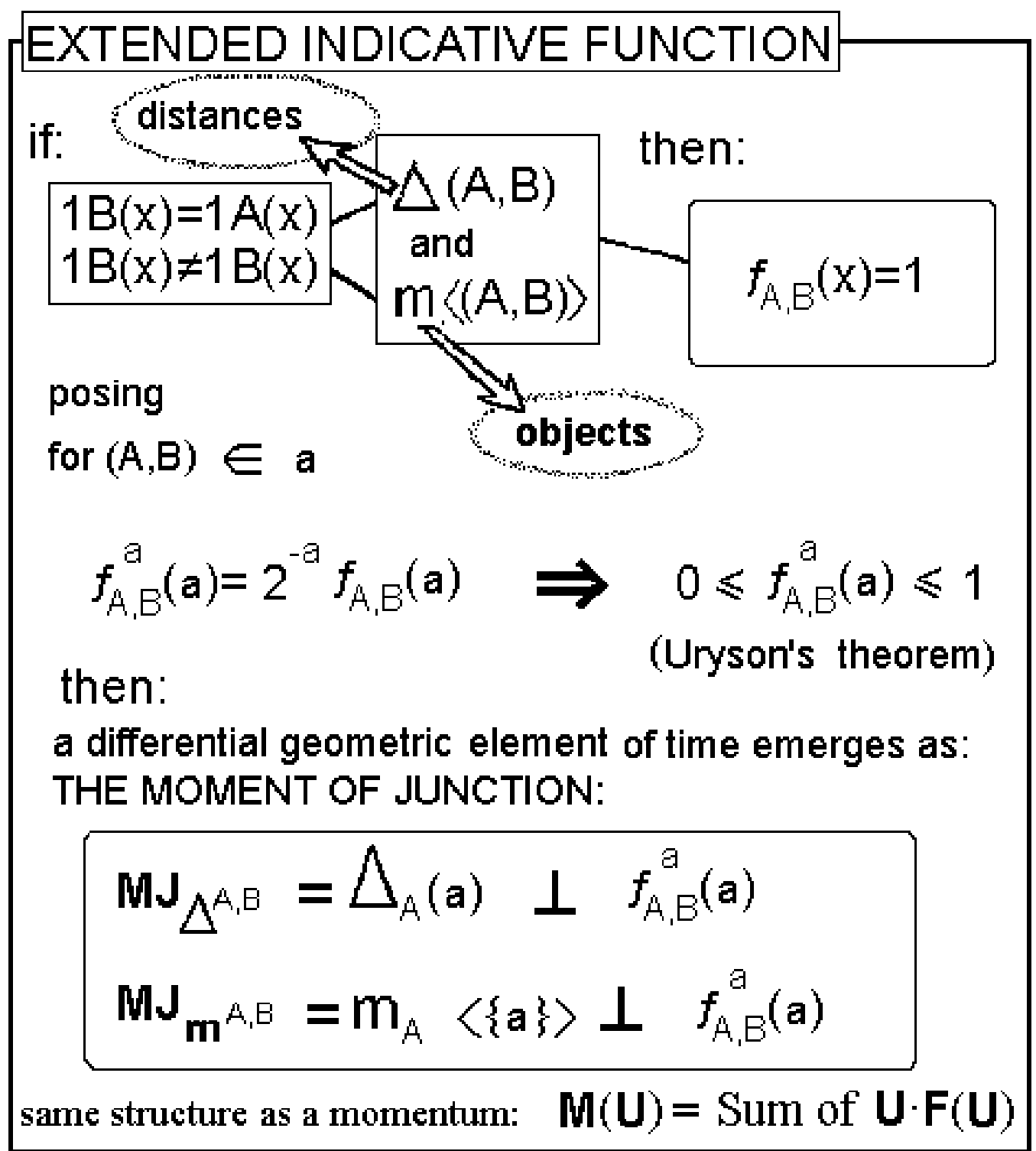

Figure 2: The composition of indicative functions of the position of points within the topological structures used as frames of references provides an extended indicative function. The composition of pairs of indicative functions provides a new function $(f)$ indicating the changes occurring in the situation of objects. The composition of the topological distances $\Delta(\mathrm{A}, \mathrm{B}, \ldots)=\complement_{\mathrm{A} \cup \mathrm{B} \ldots}(\mathrm{A} \cap \mathrm{B} \cap \ldots)$ or the topological "instances" $(\mathfrak{m}\langle(\mathrm{A}, \mathrm{B}, \mathrm{C}, \ldots)\rangle=(\mathrm{A} \cap \mathrm{B}) \cup(\mathrm{A} \cap \mathrm{C}) \cup(\mathrm{B} \cap \mathrm{C})$ with function $f$ over the populations of objects in the considered sets leads to a momentum-like structure called "Moment of Junction" and accounting for elements of the differential geometry of space. 
Then, a function $f_{(\mathrm{Ei}, \mathrm{Ei}+1)}$ (more shortly noted $\left.f_{\mathrm{E}}(\mathrm{E})\right)$ is defined as:

$$
\begin{aligned}
& f_{\mathrm{E}}=1 \text { iff }: 1_{(\mathrm{Ei})}=1_{(\mathrm{Ei}+1)}, \\
& f_{\mathrm{E}}=0 \text { iff }: 1_{(\mathrm{Ei})} \neq 1_{(\mathrm{Ei}+1)} .
\end{aligned}
$$

Summing over all points $\mathrm{x}$ in the whole of $\{\mathrm{E}\}$ provides $f_{\mathrm{E}}(\mathrm{E})$ that accounts for a distribution of the indicatrix functions of all points out of the maximum number of possibilities, which would be $2^{\mathrm{E}}$ for the set of parts of set E. This finally leads to the expression of the proportion of points involved in the mappings of parts of $\mathrm{E}(\mathrm{i}+1)$ into

$$
f_{\mathrm{E}}^{\mathrm{E}}=f_{\mathrm{E}}^{\mathrm{E}}(\mathrm{E}) / 2^{\mathrm{E}}, \quad 0<f_{\mathrm{E}}^{\mathrm{E}}(\mathrm{E})<1 .
$$

Two species of the moment of junction are represented by the composition $(\perp)$ of $f_{\mathrm{E}}^{\mathrm{E}}$ with either the set-distance of the instance, since $\mathrm{E} \supseteq \Delta(\mathrm{E}) \cup \mathfrak{m}\langle\mathrm{E}\rangle$ and the distribution of points in the complementary structures is not the complementary of their distributions. Hence:

$$
\begin{aligned}
\mathrm{MJ}_{\Delta} & =\Delta(\mathrm{E}) \perp f_{\mathrm{E}}^{\mathrm{E}}(\mathrm{E}), \\
\mathrm{MJ}_{\mathfrak{m}} & =\mathfrak{m}\langle\mathrm{E}\rangle \perp f_{\mathrm{E}}^{\mathrm{E}}(\mathrm{E}) .
\end{aligned}
$$

Generally, one will have $\mathrm{MJ}_{\Delta} \neq \mathrm{MJ}_{\mathfrak{m}}$.

As a composition of variables with their distribution, relations (3.5a,b) actually represent a form of momentum.

\subsection{Space-time as fulfilling a nonlinear convolution relation}

The "moments of junctions" (MJ) mapping an instance (a 3-D section of the embedding 4-space) to the next one apply to both the open (the distances) and their complementaries the closed (the reference objects) in the embedding spaces. But points standing for physical objects able to move in a physical space may be contained in both of there reference structures. Then, it appears that two kinds of mappings are composed with one another.

Theorem 3.2. A space-time-like sequence of Poincaré sections is a nonlinear convolution of morphisms.

Proof. The demonstration involves the following four steps.

(i) One kind of mapping $\mathcal{M}$ connects a frame of reference to the next one: here, the same organization of the reference frame-spaces must be found in two consecutive instants of our space-time, otherwise, no change in the position of the contained objects could be correctly characterized.

However, there may be some deformations of the sequence of reference frames, on the condition that the general topology is conserved, and that each frame is homeoporphic to the previous one. Mappings $\mathcal{M}$ will thus denote the corresponding category of morphisms.

(ii) The other kind of mapping $(\mathcal{J})$ connects the objects of one reference cell to the corresponding next one. Mappings $(\mathcal{J})$ thus behave as indicatrix functions of 
the situation of objects within the frames, and therefore, they are typically relevant from the "Analysis situs", that is the former name for topology, originally used by Poincaré himself (Bottazzini, 2000).

These morphisms thus belong to a complementary category.

Then, each section, or timeless instant (that is, a form of the above more general "instance") of our space-time, is described by a composition $(\bigcirc)$ of these two kinds of morphisms:

$$
\text { Space }- \text { time instant }=(\mathcal{M} \bigcirc \mathcal{J})
$$

(iii) Stepping from one to the next instant is finally represented by a mapping $\mathbf{T}$, such that the composition $(\mathcal{M} \bigcirc \mathcal{J})$ at iterate $(\mathrm{k})$ is mapped into a composition $(\mathcal{M} \perp \mathcal{J})$ at iterate $(\mathrm{k}+\mathrm{i})$ :

$$
(\mathcal{M} \perp \mathcal{J})_{\mathrm{k}+\mathrm{i}}=\mathbf{T}^{\perp}(\mathcal{M} \bigcirc \mathcal{J})_{\mathrm{k}}
$$

Hence, mapping $\left(\mathbf{T}^{\perp}\right)$ appears like a relation of the type $\mathcal{R}_{\mathrm{k}+\mathrm{i}}$ similar to that denoted below by $\mathcal{R}_{\mathrm{k}+\mathrm{j}}$ that maps a function $\mathrm{F}_{\mathrm{i}+\mathrm{k}}$ into $\mathrm{F}_{\mathrm{j}+\mathrm{k}}$ :

$$
\mathrm{F}_{\mathrm{j}+\mathrm{k}}^{\prime}=\mathcal{R}_{(\mathrm{k}+\mathrm{j})} \mathrm{F}_{\mathrm{i}+\mathrm{k}}
$$

(iv) The above relation represents a case of the generalized convolution, that is a nonlinear and multidimensional form of the convolution product, which has been first described by Bolivar-Toledo et al. (1985). The authors have proposed this concept as a tool for computing the behavior of visual perception. The demonstration that relation (3.8) is a form of a convolution is achievable by taking the following example.

Let $\alpha_{(\mathrm{j}-\mathrm{k})}$ a particular form of $\mathcal{R}_{(\mathrm{k}+\mathrm{j})}$; then equation (3.8) becomes:

$$
\mathrm{F}_{\mathrm{k}}^{\prime}=\sum \alpha_{(\mathrm{j}-\mathrm{k})} \mathrm{F}_{\mathrm{j}}
$$

that is, for the case of an integrable space

$$
\mathrm{F}^{\prime}(\mathrm{X})=\int \alpha\left(\mathrm{X}^{\prime}-\mathrm{X}\right) \mathrm{F}\left(\mathrm{X}^{\prime}\right) \mathrm{d}\left(\mathrm{X}^{\prime}\right)
$$

So the relation exhibits a great similarity with a distribution of functions, in the Schwartz sense (Schwartz, 1966):

$$
\langle f, \varphi\rangle=\sum \varphi(\mathrm{x}) f(\mathrm{x}) \mathrm{dx}
$$

or a convolution product

$$
\int_{\mathrm{E}} f(\mathrm{X}-\mathrm{u}) \mathrm{F}(\mathrm{u}) \mathrm{d}(\mathrm{u})=(f * \mathrm{~F})(\mathrm{X}) .
$$

Thus, the connection from the abstract universe of mathematical spaces and the physical universe of our observable space-time is provided by a convolution of morphisms, which supports the conjecture of relation (2.1). 
Interestingly, the distributions were primarily considered by Schwartz as an invention, in contrast with other concepts which he considered as the discovery of preexisting foundations of the total universe (Schwartz, 1997). Now, the present work could provide the distributions with the status of a discovery.

Remarks 3.2. Our observable space-time should possess nonlinear properties which necessarily involve specific features ( $\mathrm{Lin}$ and $\mathrm{Wu}, 1998$ ): therefore, peculiar approaches are needed through appropriate mathematical concepts, otherwise incorrect descriptions of the real world result as a consequence of approximate treatment of nonlinear model resolution (Wu and Lin, 2002).

\section{Relative scales in the empty-set lattice}

It has been demonstrated in Bounias and Krasnoholovets (2003) that the antifounded properties of the empty set provide existence to a lattice involving a tessellation of the corresponding abstract space with empty balls. This structure has thus been called a "tessellattice". Its formalism will be completed in section 3.2 up to Corollary 3.2.5.

\subsection{Quantum levels at relative scales}

Inside any of the above spaces, properties at micro-scale are provided by properties of the spaces whose members are empty set units. It will be shown here that particular levels of a measure of these units can be discerned.

Lemma 4.1.2. The Cartesian product of a finite beginning section of the integer numbers provides a variety of nonequal empty intervals.

Proof. Let $\mathrm{A}_{(\mathrm{N}+1)}=\{0,1,2, \ldots, \mathrm{N}\}$ denote a beginning section, that is the set of all members of a part $(M, \preceq)$ of the natural integers $(\mathbf{N})$ provided with an order relation $(\preceq)$, which are lower than $(N+1)$. A set $\{\varnothing, a, b, \ldots\}$ is equipotent with $\{0$, $1,2, \ldots, \mathrm{n}\}$ and will be denoted by $\left(\mathrm{E}_{\mathrm{n}}\right)$. Then, since any set contains $\varnothing$, one has $\left(\mathrm{E}_{\mathrm{n}}\right) \equiv \mathrm{A}_{(\mathrm{N}+1)}$.

Consider now $\left(E_{n}\right)^{2}=\left(E_{n}\right) \times\left(E_{n}\right)$. The resulting set contains ordered pairs including the diagonal (aa, bb, ...) and the heterologous pairs (ab, ac, bd, ...) accounting for rational numbers (aa $\mapsto 1$, ab $\mapsto \mathrm{a} / \mathrm{b}, \mathrm{dc} \mapsto \mathrm{d} / \mathrm{c}$, etc.). Since all members of the diagonal are mapped into 1 , there remains $n^{2}-(n-1)$ distinct pairs. A rational can be represented by a 2 -simplex or facet, whose small sides are the corresponding integers. Jumping to three dimensional conditions with $\left(\mathrm{E}_{\mathrm{n}}\right)^{3}=$ $\left(E_{n}\right)^{2} \times\left(E_{n}\right)$, each new rational is represented by a 3-simplex. This representation offers the advantage over the usual square to cube representation of avoiding several squares or cubes to share common edges or facets. Then, the number of 3-simplexes reflects the number $\mathcal{R}\left(\mathrm{E}_{\mathrm{n}}\right)^{3}$ of rational numbers available from any initial beginning segment $\mathrm{A}_{\mathrm{N}+1}$.

Consider now the cuttings or segments, represented by intervals between any two of these rational numbers. These segments represent the whole of available distances in the corresponding subpart of a 3-space. Remind that such a subpart is involved in some part of a ordered sequence, that is in a segment of an observable space-time. 
Let $[0, n]$ be the larger interval of $\left(\mathrm{E}_{\mathrm{n}}\right)$. Denote by $\left.\mathfrak{m}_{\mathrm{E}}\right] 0, \mathrm{n}[$ a measure of the open part or interior $] 0, \mathrm{n}\left[\right.$ of this interval in space $\left(\mathrm{E}_{\mathrm{n}}\right)$ : if $\mathrm{E}$ is a segment of $\mathrm{N}$, one has:

$$
\left.\mathfrak{m}_{\mathrm{E}}\right] 0, \mathrm{n}\left[=\emptyset^{\mathrm{N}} .\right.
$$

This is also encountered in the Cartesian product $\left(\mathrm{E}_{\mathrm{n}}\right)^{3}$ when it includes $(\varnothing)$.

Consider now any of the smaller intervals $(\sigma)$ in $\left(\mathrm{E}_{\mathrm{n}}\right)^{3}$ and denote by $\mathfrak{m}_{\mathrm{E}}\left[\left(\sigma\left(\mathrm{E}_{\mathrm{n}}\right)^{3}\right]\right.$ its measure. By definition:

$$
\mathfrak{m}_{\mathbb{E}}\left[\left(\sigma\left(\mathrm{E}_{\mathrm{n}}\right)^{3}\right]<\mathfrak{m}_{\mathrm{E}}[0, \mathrm{n}] .\right.
$$

Call $\varnothing^{\mathrm{Q}}$ any of the open or interior $]\left(\sigma\left(\mathrm{E}_{\mathrm{n}}\right)^{3}[\right.$, then since $\mathrm{G} \subset \mathrm{H} \Rightarrow(\mathrm{g} \subset \mathrm{G}) \subset(\mathrm{H})$ and that the distance of the interior of a set to its frontier is naught (Tricot, 1999):

$$
\emptyset^{\mathrm{Q}} \subset \varnothing^{\mathrm{N}} .
$$

This imposes an order relation holding on empty sets constructed on various segments of a finite product of finite beginning sections of the set of natural integers or equipotent to such a section.

(Q.E.D.)

Corollary 4.1.2. A finite set of rational numbers inferring from a Cartesian product of a finite beginning section of integer numbers establishes a discrete scale of relative sizes.

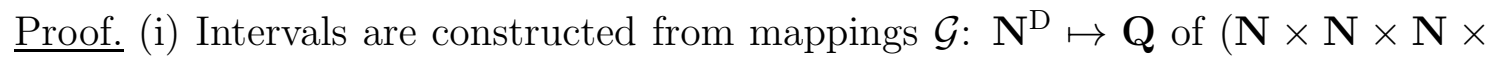
$\mathbf{N} \times \ldots)$ in $\mathbf{Q}$. For example, with $\mathbf{D}=2$, the smaller ratios available are $1 / \mathrm{n}$ and $1 /(n-1)$, so that their distance is the smaller interval: $1 /(n(n-1))$. Consider now the few smaller intervals $(\sigma)$ in $\left(\mathrm{E}_{\mathrm{n}}\right)^{3}$. One can observe, in the following order of increasing sizes:

$$
\begin{aligned}
& \forall \mathrm{n}>1 \text { : } \\
& \qquad\left(\sigma_{(\mathrm{i})}\right)=\frac{1}{\mathrm{n}^{2}(\mathrm{n}-1)}<\left(\sigma_{(\mathrm{ii})}\right)=\frac{1}{\mathrm{n}(\mathrm{n}-1)^{2}}<\left(\sigma_{(\mathrm{iii})}\right)=\frac{(\mathrm{n}-1)}{\mathrm{n}^{2}(\mathrm{n}-1)^{2}} .
\end{aligned}
$$

Let us consider maximal of the ratios of larger $(n)$ to smaller $\left(1 / n^{2}(n-l)\right)$ segments; then one gets

$$
\frac{\max (\sigma)}{\min (\sigma)}=\mathrm{n}^{3}(\mathrm{n}-\mathrm{l})
$$

One possibility of a scaling progression covering integer subdivisions (n) consists in dividing a fundamental segment $(\mathrm{n}=1)$ by 2 , then each subsegment by 3 , etc. Thus the size of structures is a function of iterations (n). At each step $\left(\nu_{\mathrm{j}}\right)$ the ratio of the size in the dimension $\mathrm{D}$ will be $\left(\Pi \nu_{\mathrm{j}}\right)^{\mathrm{D}}$, so that the maximal ratio will be, following (4.5),

$$
\varrho \propto\left\{\left(\Pi \nu_{\mathrm{j}}\right)^{\mathrm{D}}\left(\Pi \nu_{\mathrm{j}}-1\right)\right\}_{\mathrm{j}=1 \rightarrow \mathrm{n}} .
$$

The manifold $\left(\Pi \nu_{\mathrm{j}}\right)$ is a commutative Bourbaki-multipliable indexed on the integer section $I=[1, n]$. In practice, values can be written as $\varrho_{j}=a_{j} \cdot 10^{x_{j}}$ where in the base 10 one will take $a_{j}$. belonging to the (always existing) neighborhood of unity, i.e. $\left.a_{j} . \in\right] 1\left[\right.$, and look at the corresponding integer exponents $x_{j}$ as the order of sizes of structures constructed from the lattice $\mathcal{L}=\left(\Pi \nu_{\mathrm{j}}\right)^{\mathrm{D}}$. Regarding distances 
$(\mathrm{D}=1)$ to areas and volumes $(\mathrm{D}=2$ and 3$)$ equation (4.6) consistently provides the predicted orders of scales listed in Table 1. The latter represent quantic-like levels of clusters of objects sharing successive orders of sizes while constructed one from the others.

(ii) There is a finite number of segments or intervals, since their supremum is the number of 3-simplexes, which is finite, and contains redundant terms. This number is the number of pairwise combinations of distinct rationals. Therefore:

$$
\sup \mathcal{N}\left(E_{n}\right)^{3}=C_{n^{2}-\mathrm{n}+1}^{2}=\frac{1}{2} \mathrm{n}(\mathrm{n}-\mathrm{l})\left(\mathrm{n}^{2}-\mathrm{n}+1\right) .
$$

A general formula for exactly $\left(\mathcal{N}\left(\mathrm{E}_{\mathrm{n}}\right)^{3}\right)$ is not readily available since it involves prime numbers occurring in $\left(\mathrm{E}_{\mathrm{n}}\right)$, but this does not change the meaning of the reasoning.

Finally, there is a finite number of ratios of segment sizes imposing on a subpart of a space-time sequence a limited number of relative scales for any of the objects represented by closed subspaces in $\left(\mathrm{E}_{\mathrm{n}}\right)^{3}$.

(Q.E.D.)

Corollary. The axiom of availability (stating that a rule provided to a set must be considered as explicitly applying to the set's members and parts) is necessary to an exploration of an unknown space, either mathematical or physical.

The simple following counter-example provides the proof. Suppose the axiom of availability is not stated: then, a complete subset of the rational numbers may not be provided in all bases by the Cartesian product of a segment of natural numbers.

Let $\mathrm{E}_{\mathrm{n}}=\{1,2, \ldots, \mathrm{n}\}$ with $\mathrm{n}<9$, and let two integers, $\mathrm{p}, \mathrm{q} \in \mathrm{E}_{\mathrm{n}}$. Then, the pair $(p, q) \in\left(E_{n}^{2}\right)=E_{n} \times E_{n}$. Usually, $(p, q)$ accounts for the ratio $p / q$, so that the set of pairs $(p, q)$ is equipotent to the set of rational numbers noted as fractions: $\left(e_{i} \cdot d_{1} d_{2} \ldots d_{i}\right)$ where $e_{i}$. stands for the entire part and $d_{1} d_{2} d \ldots d_{i} \ldots$ for the decimal part.

Let $\mathrm{n}=4$, and take $\mathrm{p}=1, \mathrm{q}=4$. Then the ordered pair $(1,4)$ stands for the ratio $1 / 4$. However, writing $1 / 4=0.25$ needs digit 5 to be available, whereas one has just $1,2,3,4$ available, not 5 . Therefore, in this system, since digit 5 does not exist unless the additional axiom of the addition is introduced, the mapping of ordered pairs to the writing in base 10 of the corresponding rational numbers is not valid. The availability of the power set of parts, i.e. the infinitely iterated sets of parts of the sets of parts (Bounias, 2001) is enough to break this barrier.

Table 1. Range and intermediate levels of the scale of size of objects composing a universe constructed as described in relation (4.6). Intervals constructed with powers of 10 on the neighborhood of unity (a]1[) are confronted through dimensions: (i) $\mathrm{D}=1$ with the simple multipliable set $\Pi_{\mathrm{j}}\left(\nu_{\mathrm{j}}\right)$; (ii) $\mathrm{D}=2$ involving intervals $\mathrm{l} / \mathrm{n}(\mathrm{n}-\mathrm{l})$; (iii) $\mathrm{D}=3$ involving intervals $1 / \mathrm{n}^{2}(\mathrm{n}-\mathrm{l})$. Here the choice $] 0.7,1.3[$ just reflects the case of a normal distribution quantile sufficiently close to unity as the mean.

Notes: $\left(^{*}\right)$ and $(* *)$ suggest further levels of higher scale universes; $(* * *)$ a continued cluster from $10^{142}$ to $10^{171}$, suggesting a quite different organization of matter (in the case of $\left(^{*}\right)$, $\mathrm{x}$ varies from 82 to 120 ; in the case of $\left(^{* *}\right), \mathrm{x}=139$; in the case of $(* * *)$, x changes from 142 to 171). Predictable orders of size, from the Planck scale, roughly comply with quark-like size $\left(10^{10-11}\right)$, particle to atoms $\left(10^{11-17}\right)$, molecules $\left(10^{21}\right)$, human size $\left(10^{28}\right)$, stars and solar systems $\left(10^{40-42}\right)$, up to the estimated upper limit $\left(10^{56}\right)$ which could be bounded by a "anti-Planck" scale at $\left(10^{60-61}\right)$. 


\begin{tabular}{|c|c|c|c|c|c|c|}
\hline \multicolumn{3}{|c|}{$\rho=\Pi_{\mathrm{j}}\left(\nu_{\mathrm{j}}\right)$} & \multicolumn{2}{|c|}{$\begin{array}{c}\rho= \\
\left\{\Pi_{\mathrm{j}}\left(\nu_{\mathrm{j}}\right) \times\left(\Pi_{\mathrm{j}}\left(\nu_{\mathrm{j}}-1\right)\right)\right\}\end{array}$} & \multicolumn{2}{|c|}{$\begin{array}{c}\rho= \\
\left\{\left(\Pi_{\mathrm{j}}\left(\nu_{\mathrm{j}}\right)\right)^{2} \times\left(\Pi_{\mathrm{j}}\left(\nu_{\mathrm{j}}-1\right)\right)\right\}\end{array}$} \\
\hline $\mathrm{x}$ & $\begin{array}{l}(1 \pm 0.3) \\
\times 10^{\mathrm{x}}\end{array}$ & $(\nu)$ & $\begin{array}{l}1 \pm 0.3) \\
\times 10^{\mathrm{x}}\end{array}$ & $(\nu)$ & $\begin{array}{l}1 \pm 0.3) \\
\times 10^{\mathrm{x}}\end{array}$ & $(\nu)$ \\
\hline 0 & $1 \times 10^{0}$ & (1) & 0 & (1) & 0 & \\
\hline 2 & $1.20 \times 10^{2}$ & (5) & 0 & $(1)$ & 0 & \\
\hline 10 & $0.87 \times 10^{10}$ & (14) & & & & \\
\hline 11 & & & & & $1.28 \times 10^{11}$ & (7) \\
\hline 17 & $1.22 \times 10^{17}$ & (19) & & & & \\
\hline 21 & $1.12 \times 10^{21}$ & (21) & & & & \\
\hline 28 & $1.09 \times 10^{28}$ & $(27)$ & & & & \\
\hline 29 & & & $1.27 \times 10^{29}$ & (17) & & \\
\hline 31 & $0.88 \times 10^{31}$ & (29) & & & & \\
\hline 33 & $0.87 \times 10^{33}$ & (33) & & & & \\
\hline 40 & $1.03 \times 10^{40}$ & $(35)$ & & & $0.91 \times 10^{40}$ & (16) \\
\hline 42 & & & $1.26 \times 10^{42}$ & $(22)$ & & \\
\hline 56 & $1.20 \times 10^{56}$ & $(45)$ & $1.18 \times 10^{56}$ & (27) & & \\
\hline 59 & & & $0.93 \times 10^{59}$ & $(28)$ & $1.33 \times 10^{59}$ & $(21)$ \\
\hline 61 & $1.24 \times 10^{61}$ & & & & & \\
\hline$*$ & & $*$ & $*$ & $*$ & $*$ & $*$ \\
\hline 82 & $0.83 \times 10^{82}$ & $(60)$ & & & & \\
\hline 84 & & & & & $1.29 \times 10^{84}$ & $(27)$ \\
\hline 99 & & & $1.12 \times 10^{99}$ & & & \\
\hline 100 & $1.20 \times 10^{100}$ & $(70)$ & & & & \\
\hline 112 & & & $1.25 \times 10^{112}$ & $(45)$ & & \\
\hline 115 & $1.13 \times 10^{115}$ & & & & & \\
\hline 117 & $0.89 \times 10^{117}$ & (79) & & & & \\
\hline 120 & & & & & $1.10 \times 10^{120}$ & $(35)$ \\
\hline$* *$ & $* *$ & $* *$ & $* *$ & $* *$ & $* *$ & $* *$ \\
\hline $\begin{array}{l}139 \\
* * *\end{array}$ & & & $* * *$ & * * * & $0.85 \times 10^{139}$ & $(39)$ \\
\hline 142 & 1014 & $* * *$ & $* * *$ & $* * *$ & $* * *$ & $* * *$ \\
\hline $\begin{array}{l}142 \\
150\end{array}$ & $\begin{array}{l}1.25 \times 10^{2} \\
100 \times 10^{150}\end{array}$ & $\begin{array}{l}(92) \\
(96)\end{array}$ & & & & \\
\hline 163 & $1.00 \times 10^{163}$ & (103) & & & & \\
\hline 171 & $1.24 \times 10^{171}$ & (107) & $0.92 \times 10^{171}$ & $(62)$ & & \\
\hline
\end{tabular}

\subsection{About boundaries}

Converging sequences of rational numbers are known to provide the set of real numbers. However in a space of finite dimension, real numbers cannot infer by this way. In contrast, infinitely descending sequences (in the Mirimanoff sense) of pairs of the $\varnothing^{\mathrm{Q}}$ and $\varnothing^{\mathrm{N}}$ types can be found inside each part $\{\varnothing\}$. Therefore, infinitely 
smaller intervals could always be found in the lower range of scales. These infinitely decreasing sizes are of a different nature in that they fill each discrete part $\{\varnothing\}$. However, this does not mean that these structures necessarily are the ultimate ones. Besides the empty set as the set with no members, one has :

Proposition 4.2.1. There exists a set with no parts.

Preliminary proof. The space of $(\varnothing)$ provided with the complementarity property gives raise to abstract sets equipotent to sets of numbers, so that for instance $\left(\mathrm{W}^{\mathrm{m}}\right)$ owns parts equipotent to $\mathbf{R}^{\mathrm{m}}$. Consider the union $\mathrm{U}=\mathrm{U}(\mathrm{W})$ of all possible $\mathrm{W}^{\mathrm{m}}$ spaces, and its structural complementary in the resulting fundamental space $\mathrm{F}(\mathrm{U})$ : the structural complementary of a space with parts is a space with no parts. Since it has no parts it cannot have members. In effect, if its members were nonempty, this would be a nonempty space, which is excluded by definition, while if it had empty parts, it would just be $\varnothing$. Thus, there exists a set denoted here by $(\varnothing)$ that has neither members nor parts. Furthermore, is $\not$ contained in none of existing sets: otherwise it would be the complementary of Borel sets and therefore it would include parts of itself. This provides the set of possible structures with a lower boundary.

The fundamental set embedding space $U$ can thus be written:

$$
\mathrm{F}(\mathrm{U})=\{\cup(\mathrm{W})\} \cup(\not) .
$$

In particular, given a partition of $\cup(W)$ into $W_{X}$ and $W_{Y}$, the separating distance between $W_{X}$ and $W_{Y}$ in $\cup(W)$ is naught iff it does not belong to the filter $\mathcal{F}$ holding on $W$, that is since $\not$ and only $\not \in \mathcal{F}$ :

$$
\Delta_{\cup(\mathrm{W})}\left(\mathrm{W}_{\mathrm{X}}, \mathrm{W}_{\mathrm{Y}}\right)=(\not) \text {. }
$$

The empty hyperset can no longer be treated as it was formerly considered the well founded empty set: in particular, $\varnothing \notin \mathcal{F}$ whereas $\varnothing^{\varnothing}$ has not the same status.

Corollary 4.2.1. The set with neither member nor parts is strictly unique.

Proof. Let two universes $\mathrm{F}(\mathrm{Ui})=\{\cup(\mathrm{Wi})\} \cup(\not \mathbf{i})$ and $\mathrm{F}(\mathrm{Uj})=\{\cup(\mathrm{Wj})\} \cup(\not \mathbf{j})$ as in (3.8). Then, $(\not \mathbf{\phi} \mathbf{i}) \cup(\boldsymbol{\phi} \mathbf{j})=(\not \boldsymbol{\phi})$ since the reunion of no parts and no member is just identical with (0). However this would also allow one to write: $(\not{\phi})=\{(\not \mathbf{j}),(\not \mathbf{c j})\}$ and $(\not \mathbf{j})$ would be composed of two parts, which is contradiction.

Set $(\not \subset)$ can thus be denoted as the "nothingness singleton" $\{\not \boldsymbol{\phi}\}$.

Corollary 4.2.2. A set equipotent to the set of natural integers $\mathbf{N}$ is intrinsically of nonzero measure, and a segment $\mathrm{E}$ of $\mathbf{N}$ cannot be of measure naught even relatively to the correspondingly available segment of $\mathbf{Q}$. A member of $\mathbf{N}^{\mathbf{x}}$ is never of measure naught.

Preliminary proof. (i) given $\mathbf{N}$ and only $\mathbf{N}$ as the fundamental set, one cannot insert each member in an interval as small as needed, since there exists no segment available with size lower than 2 units, able to contain each point. For this to be achieved it is necessary to provide the system with at least $\mathbf{N} \times \mathbf{N}$ so as to generate $\mathbf{Q}$. The former case is called the intrinsic measure on $\mathbf{N}$, while the latter is the measure on $\mathbf{N}$ relatively to $\mathbf{Q}$.

(ii) Let $\mathrm{E}(\varnothing)$ be equipotent to a beginning section of $(\mathbf{N})$ : the result presented above states that there exists a finite number of rational inferring from the cartesian 
product of this space. Therefore it is not possible to insert the members of $\mathrm{E}$ in a sum of intervals of $\mathrm{E} \times \mathrm{E}$ as small as needed. Thus $\mathrm{E}(\varnothing)$ can be of neither intrinsic nor Q-relative measure naught.

(iii) Let finally $\mathrm{E}^{\mathrm{x}}=(\mathbf{N} \times \mathbf{N} \times \ldots \times \mathbf{N})_{\text {finitely } \mathrm{x} \text { times, } \mathrm{x}<1}$ : each member of the equipotent set is an ordered pair. Even if an unordered $N$-uple $\{a, b, \ldots\}$, whatever the nature of a and b, could be eventually of measure zero, excepted intrinsically, an ordered $\mathrm{N}$-uple (ab...) owns a dimension $\geq 1$ and cannot be inserted in an interval as small as needed.

Corollary 4.2.3. A finite set is not of measure zero if its contains members and parts that are representative of a space of dimension $\mathrm{D}>1$.

Corollary 4.2.4. Due to uniqueness of $(\not)$, the "tessellattice" is correctly tessellated since no gap can subsist between any two or more of its empty tessellation balls. Furthermore, $\not$ provides the tessellattice with a infinum, and thus with a partial order.

\section{Particles in a lattice universe}

\subsection{Introduction}

Let space be represented by the lattice $\mathrm{F}(\mathrm{U})=\cup(\mathrm{W}) \cup \not$ as from relation (9.1) in Bounias and Krasnoholovets (2002), where $\not$ is the set with neither members nor parts. This accounts for both relativistic space and quantic void, since: (i) the concept of distance and the concept of time have been defined on it, and (ii) this space holds for a quantum void since on one hand, it provides a discrete topology, with quantum scales, and on the other hand it contains no "solid" object that would stand for a given provision of physical matter.

The above relation (3.2) involves the mapping of a frame of reference into its image frame of reference in the next section of space-time. Without such a continuity there would be no possibility of assessing the motion of any object in the perceived universe. This is exactly a case of "analysis situs", in the original meaning used by Poincaré. Now, continuity in the perception of a space-time is provided iff the frames of references are conserved through homeomorphic mappings. This means that there is no need for exact replication: just topological structures should be conserved. Hence, the realization of varieties if allowed, even in a space of different dimension. This supports the following:

Proposition 5. The sequence of mappings of one into another structure of reference (e.g. elementary cells) represents an oscillation of any cell volume along the arrow of physical time.

However, there is a case in which a threshold may exist, precluding the conservation of homeomorphisms: let a transformation of a cell involving some iterated internal similarity (see Figure 3 for simplified example). Then, if $\mathrm{N}$ similar figures with similarity ratios $1 / \mathrm{r}$ are obtained, the Bouligand exponent (e) is given by

$$
\mathrm{N}(1 / \mathrm{r})^{\mathrm{e}}=1
$$

and the image cell gets a dimensional change from $d$ to $d^{\prime}=\ln (\mathrm{N}) / \ln (\mathrm{r})=\mathrm{e}>\mathrm{l}$. 

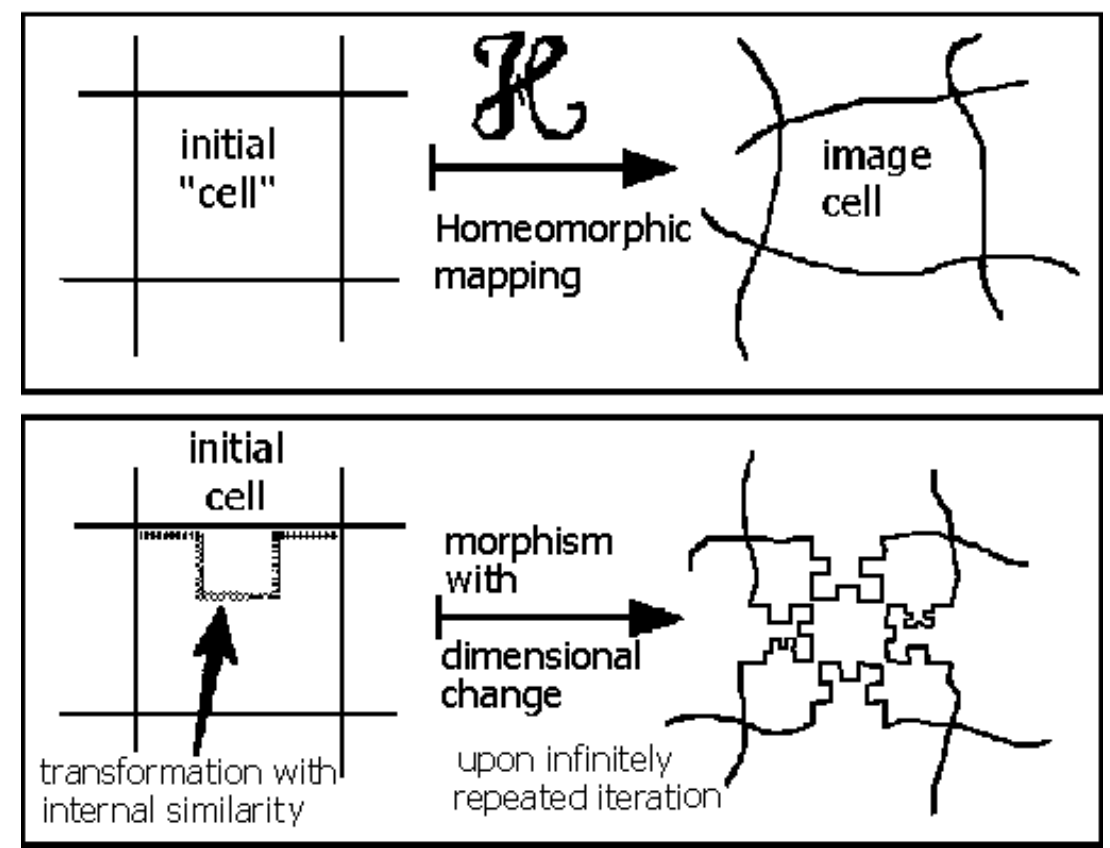

Figure 3: The continuity of homeomorphic mappings of structures is broken if once a deformation involves an iterated transformation with internal self-similarity, which involves a change in the dimension of the mapped structure. Here, various the first 2 or 3 steps of the iteration are sketched, with basically the new figure jumping from (D) to approximately $(\mathrm{D}+1.45)$. The mediator of transformations is provided in all cases by empty set units.

Then, the putatively homeomorphic part of the image cell is no longer a continued figure and the transformed cell no longer owns the property of a reference cell.

This transformation stands for the formation of a "particle" also called "particled cell" or more appropriately "particled ball", since it is a kind of topological ball $\mathrm{B}[\varnothing, \mathrm{r}(\varnothing)]$. Thus the following:

Statement 5. A particled ball is represented by a nonhomeomorphic transformation in a continuous deformation of space elementary cells.

\subsection{On quanta of fractality and fractal decomposition}

Before examining the interactions of particled balls with the degenerate space lattice and further with other particled balls, it is necessary to demonstrate some mathematical preliminaries.

\subsubsection{Quanta of fractality}

A minimum fractal structure is provided by a self-similar figure whose combination rule includes a initiator and a generator, and for which the similarity dimension exponent is higher than unity.

(i) Initiator. Due to self-similarity of $\geq \varnothing$, each time one considers the complementary of itself in itself, one gains: $\varnothing \mapsto\{(\varnothing), \varnothing\}$. That is, one ball gives two identical balls. This is continued into a sequence of $\{1 / 2,1 / 4, \ldots, 1 / 2 \mathrm{n}\}$ numbers 
at the $\mathrm{n}^{\text {th }}$ iteration. Thus, the series $(\mathrm{I})=\sum_{\mathrm{i}=1 \rightarrow \infty}\left\{1 / 2^{\mathrm{i}}\right\}$ stands for the initiator, providing the needed iteration process. The terms of (I) are indexed on the set of natural numbers, and thus provide an infinitely countable number of members.

Interestingly, $2^{\mathrm{n}}$ also denotes the number of parts from a set of $\mathrm{n}$ members,

(ii) Generator. Let an initial figure (A) be subdivided into $r$ subfigures at the first iteration. The similarity ration is thus $\varrho=1 / \mathrm{r}$. Let $\mathrm{N}=(\mathrm{r}+\mathrm{a})$ be the number of subfigures constructed on the original one. Then, one has $e=\operatorname{Ln}(r+a) / \operatorname{Ln} N$. The value of e is bounded by unity if $\mathrm{r}$ is extended to infinity. For any $\mathrm{r}$ finite (likely the case in a physical world), the exponent e is above unity. Then:

$$
\{\min (\mathrm{e}) \mid \mathrm{e}>1\}=\operatorname{Ln}(\max (\mathrm{r})+1) / \operatorname{Ln}(\max (\mathrm{r})) \text {. }
$$

This completes the description of a quantum of fractality.

\subsubsection{The fractal decomposition principle}

Let a fractal system be denoted by $\Gamma$, such as $\Gamma=\{(\varnothing),(r+a)\}$. More complex systems just need to be incorporated several different subfigures to which the following reasoning could be extended. At the $\mathrm{n}^{\text {th }}$ iteration, the number of additional subfigures is $\mathrm{N}_{\mathrm{n}}=(\mathrm{r}+\mathrm{a})^{\mathrm{n}}$. The similarity ration becomes $\varrho_{\mathrm{n}}=1 / \mathrm{r}^{\mathrm{n}}$. Owing to subvolumes $\left(\mathrm{v}_{\mathrm{i}}\right.$ constituted at each iteration, in the simplest case $\mathrm{v}_{\mathrm{i}}=\mathrm{v}_{\mathrm{i}-1}(1 / \mathrm{r})^{3}$. Since at the $\mathrm{i}^{\text {th }}$ iteration as many as $\mathrm{N}_{\mathrm{i}}=(\mathrm{r}+\mathrm{a})^{\mathrm{i}}$ such subvolumes are created, the total volume occupied by the subvolumes formed by the fractal iteration to infinity is the sum of the series:

$$
\mathrm{v}_{\mathrm{f}}=\sum_{(\mathrm{i}=1 \rightarrow \infty)}\left\{(\mathrm{r}+\mathrm{a})^{\mathrm{i}} \cdot \mathrm{v}_{\mathrm{i}-1}(1 / \mathrm{r})^{3}\right\}
$$

that can be developed into

$$
\mathrm{v}_{\mathrm{f}}=\sum_{(\mathrm{i}=1 \rightarrow \infty)}\left\{\left[\Pi_{(\mathrm{i}=1 \rightarrow \mathrm{n}}\right](\mathrm{r}+\mathrm{a})^{\mathrm{i}-1} /(\mathrm{r})^{3 \mathrm{i}}\right\}
$$

This leads to the following definition:

Definition 5.2. A fractal decomposition consists in the distribution of the members of the set of fractal subfigures:

$$
\Gamma \supset\left\{\sum_{\mathrm{i}=1 \rightarrow \infty}\left\{(\mathrm{r}+\mathrm{a})^{\mathrm{i}} \cdot \mathrm{v}_{\mathrm{i}-1}(\mathrm{l} / \mathrm{r})^{3}\right\}\right\}
$$

constructed on one figure, among a number of connected figures $\left(\mathrm{C}_{1}, \mathrm{C}_{2}, \ldots, \mathrm{C}_{\mathrm{k}}\right)$ similar to the initial figure $(\mathrm{A})$. If $\mathrm{k}$ reaches infinity, then all subfigures of $\mathrm{A}$ are distributed and (A) is no longer a fractal.

Figure 4 shows in a very schematic way the fractal decomposition process.

Corollary 5.2. Reciprocally, a fractal figure can be recomposed from an infinitely enumerable set of self-similar figures whose numbers and sizes are distributed as in relation $(5.2)-(5.2 \mathrm{a})$. 


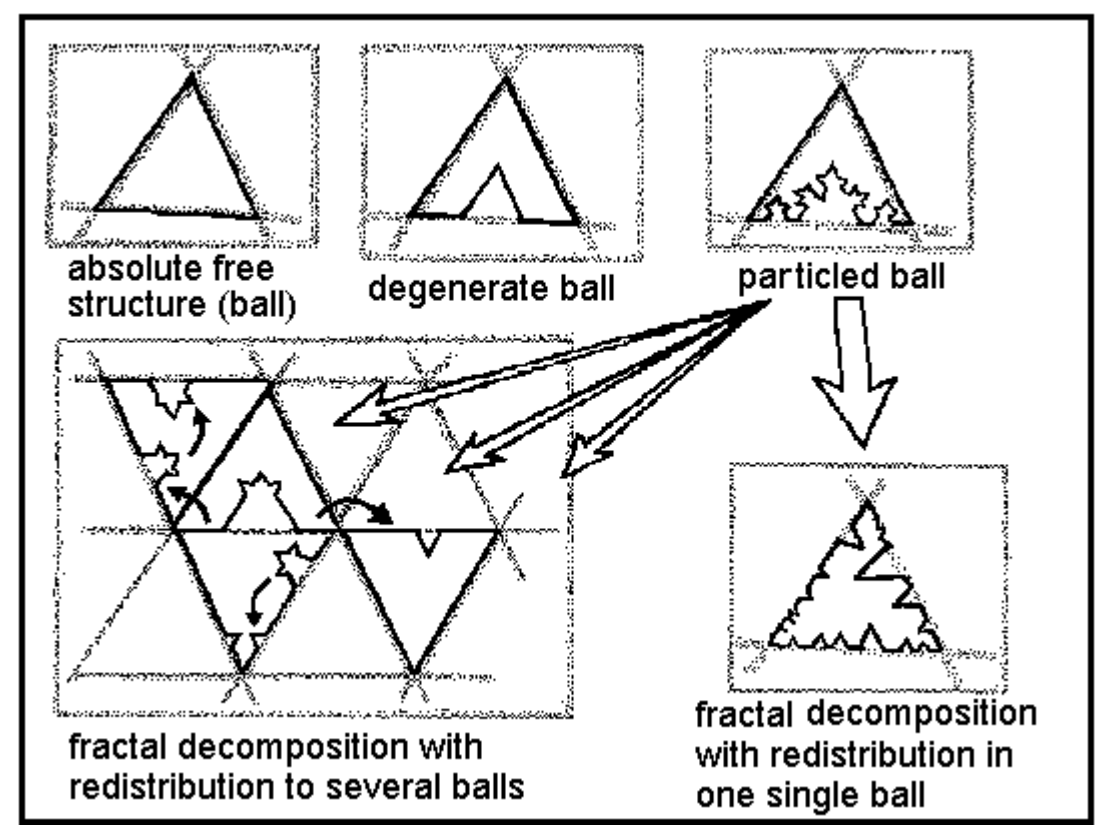

Figure 4: A canonical ball is represented as a triangle, figuring 3 dimensions, in a metaphorical form. A degenerate ball keeps the same dimension, in contrast with a particled ball endowed with a fractal substructure. A complete decomposition into one single ball $(\mathrm{k}=1)$ conserves the volume without keeping the fractal dimension. The von Kochlike fractal has been simplified to 3 iterates for clarity.

\subsection{Interactions involving exchanges of structures}

\subsubsection{Scattering from friction}

Let a ball (A) containing a fractal subpart on it, as shown in Figures 3 and 4. Deformations can be transferred from one to another ball with conservation of the total volume of the full lattice (which is constituted by a higher scale empty set). If a fractal deformation is subjected to motion, it will collide with surrounding degenerate balls. Such collisions will result in fractal decompositions at the expense of (A) whose exponent $\left(\mathrm{e}_{\mathrm{A}}\right)$ will decrease, and to the profit of degenerate cells: if $\mathrm{k}$ is finite, one will have $\left(e_{i}\right)>1,\left(e_{2}\right)>1, \ldots,\left(e_{k}\right)>1$. A fractal decomposition gives raise to a distribution of coefficients $f\left(e_{k}\right)$, whose most ordered form is a sequence of decreasing values:

$$
f\left(\mathrm{e}_{\mathrm{k}}\right)=\left\{\left(\mathrm{e}_{\mathrm{i}}\right)_{(\mathrm{i} \in \mathrm{k}, 1])}\right\}
$$

\subsubsection{Boundary conditions}

From relation (5.4), it follows that the remaining of fractality decreases from the kernel (i.e. the area adjacent to the original particled deformation) to the edge of the inerton cloud. At the edge, it can be conjectured that, depending on the local resistance of the lattice, the last decomposition (denoted as the $\mathrm{n}^{\text {th }}$ iteration) can result in $\left(e_{n}\right)=1$. Since in all cases one has $\left(e_{n-1}>1\right.$, eventhough the corresponding remaining deformation is a fragment of the original fractal structure, then the resulting non-fractal deformations can be theoretically distributed up to infinite 
distance.

Therefore, while central inertons exhibit decreasing higher boundaries, edge inertons are bounded by a rupture of the remaining fractality.

\subsection{Discussion and alternative hypothesis}

\subsubsection{About infinitesimal elements}

One could wonder whether an infinite iteration could not physically take a infinite time and thus would be non-physical, (i) One answer is that all from the first iteration would take a time decreasing to infinitely small values to occur, (ii) Another would state that a quantum of fractality with the simplest geometrical generator and initiator would also stand for a quantum of time corresponding with the maximum velocity for the corresponding particle, and that the velocity of a nonfractal deformation would stand for the maximum velocity of non-massive corpuscles.

\subsubsection{Incomplete fractality hypothesis}

However, one could also conjecture that mass could be in some way proportional to the number of iterations on the way to a fractal whose completion would be only a theoretical (likely hyperbolic) limit. Here again, the number of iterations provide an alternative kind of qualitative jump, where $n=1$ iteration would stand for non-massive corpuscle, and $\mathrm{n}>2$ for massive particles.

\subsubsection{Topological alternatives}

Some other features about massive versus non-massive properties remain to be explored. Among other candidates, one could list dense versus non-dense, compact versus not compact, complete versus not complete subspaces. Conjecturally, one could envision that the space of particles could be everywhere dense in that of superparticles while non-dense in the total space, and the space of superparticles could be dense in the total space. This is matter of work in progress.

\section{Practical predictions from the inertons theory}

\subsection{Preliminaries}

A particled ball as described above provides a formalism describing the elementary particles proposed by Krasnoholovets and Ivanovsky (1993), Krasnoholovets $(1997 ; 2000)$. In this respect, mass is represented by a fractal reduction of volume of a ball, while just a reduction of volume as in degenerate cells is not sufficient to provide mass. Accordingly, if $\mathrm{v}_{\mathrm{o}}$ is the volume of an absolutely free cell, then the reduction of volume resulting from a fractal concavity is the following: $\mathrm{V}^{\text {part }}=\mathrm{v}_{\mathrm{o}}-\mathrm{v}_{\mathrm{f}}$, that is, according to relation $(5.3 \mathrm{~b})$ :

$$
\mathrm{V}^{\mathrm{part}}=\mathrm{v}_{\mathrm{o}} \cdot\left(1-\sum_{(\mathrm{i}=1 \rightarrow \infty)}\left\{\left[\Pi_{(\mathrm{i}=1 \rightarrow \mathrm{n})}(\mathrm{r}+\mathrm{a})^{\mathrm{i}-1}\right] /(\mathrm{r})^{3 \mathrm{i}}\right\}\right)
$$


that is, since $(r+a)=(r)^{e}$, we have instead of $(6.1 \mathrm{a})$

$$
\mathrm{V}^{\mathrm{part}}=\mathrm{v}_{\mathrm{o}} \cdot\left(1-\sum_{(\nu)}\left(\sum_{(\mathrm{i}=1 \rightarrow \infty)}\left\{\left[\Pi_{(\mathrm{i}=1 \rightarrow \mathrm{n})}\left(\mathrm{r}_{\nu}\right)^{\mathrm{e}_{\nu}(\mathrm{i}-1)}\right] /\left(\mathrm{r}_{\nu}\right)^{3 \mathrm{i}}\right\}\right)_{\nu}\right)
$$

where $(\nu)$ denotes the possibly several fractal concavities affecting the particled ball. This point will be the matter of an examination of the various kinds of particles predicted by the model in Part 3 of this study.

Relation $(6.1 \mathrm{~b})$ relates the volume of particled balls to the fractal dimensional change (e), which can be expressed as the following:

Proposition 6.1. The mass Ma of a particled ball A is a function of the fractalrelated decrease of the volume of the ball:

$$
\mathrm{Ma} \propto\left(1 / \mathrm{V}^{\text {part }}\right) \cdot\left(\mathrm{e}_{\mathrm{v}}-1\right) \mathrm{e}_{\mathrm{v}}>1
$$

where (e) is the Bouligand exponent, and $(e-1)$ the gain in dimensionality given by the fractal iteration.

Just a volume decrease is not sufficient for providing a ball with mass, since a dimensional increase is a necessary condition.

\subsection{Foundations of the inertons theory}

Two interaction phenomena are considered: first, the elasticity $(\gamma)$ of the lattice favors an exchange of fragments of the fractal structure between the particled ball and the surrounding degenerate balls. In a first approach, the resulting oscillation has been considered homogeneous. Second, if the particled ball has been given a velocity, its fractal deformations collide with neighbor degenerate balls and exchanges of fractal fragments occur.

Proposition 6.2. The velocity of the transfer of deformations is faster for non fractal deformations and slower for fractal ones, at slowering rates varying as the residual fractal exponent $\left(e_{i}\right)$.

Justifications. A fractal subvolume owns an infinite surface, which imposes a more important transfer than the progression of a non fractal volume, which involves a finite surface. More generally each iteration step involves a proper quantum of transfer time.

Proposition 6.3. The motion of the system constituted by a particled ball and its inerton cloud provides the basis for de Broglie and Compton wavelengthes.

Justifications. During the progression of a particled ball, its mass is progressively transferred to a cloud of surrounding balls which get fragments of the particle mass. These new quasi-particles are called "inertons". The inertons velocity is faster due to lower fractal dimension, and the cloud migrates forwards up to the state where the residual mass of the particle is low enough. At this step, the particle has progresively lost its velocity due to the collisions: then, since collision with the degenerate lattice balls stop, no more inertons are produced. At this time, the elasticity of the lattice starts to reinject the fragments of deformations and progressively restore the initial fractal in the particled ball, which is reconstructed. Similarly, the equivalent in 
momentum (that is $(\langle m\rangle \cdot\langle v\rangle$ ), where $\langle m\rangle$ and $\langle v\rangle$ respectively denote the average mass and velocity of the system composed with \{particle + inertons and further denoted $(\Xi)$ is progressively retransfered to the particled ball, which gets back its initial velocity.

Then, the trajectory of a particle is represented by a complex oscillating system which drives from a state (denoted as initial) where the particle owns its full mass and velocity to a state where its velocity is minimal (eventually null) and part of its mass has been transferred to the inerton cloud (Table 2).

This represents a cycle with a period $\lambda$ of the path, such that $\min \left\{M, V^{\text {part }}\right\}$ occurs at $\lambda / 2$ : this defines a parameter identified with the de Broglie wavelength. Then, the system $(\Xi)$ which exhibits similarities with a crystallite has an oscillating size called $\widetilde{\lambda}_{v_{0}}$ and identified with the Compton wavelength (Krasnoholovets, 1997, $2000,2001)$. The inertons cloud itself is characterized by a specific amplitude $\Lambda$ that has been related with the other two ones by the following relations:

$$
\Lambda=\lambda \hat{c} / v_{\mathrm{o}}=\widetilde{\lambda}_{v_{\mathrm{o}}} \cdot \hat{c}^{2} / v_{\mathrm{o}}^{2}
$$

where $v_{\mathrm{o}}$ is the initial velocity of the particled ball and $\hat{c}$ is the velocity of emitted inertons.

Table 2 summarizes the main steps and features of the particle to inertons cycle.

Remark 6.1. The center of mass of the system $(\Xi)$ permanently oscillates between the original particle and the inerton cloud. This introduces an additional parameter whose various forms provide a solid support to the concept of spin (Krasnoholovets, 2000).

Remark 6.2. The system composed with the particle and its inertons cloud is not likely to be of homogeneous shape. Therefore, their relative motion will expectably exhibit an eddy-like form (Wu and Lin, 2002). This property will be accounted for in spin-related properties of observable matter.

Proposition 6.4. The fractality of particle-giving deformations gathers its space parameters $(\varphi \mathrm{i}) \mathrm{i}$ and velocities $(\mathfrak{v})$ into a self-similarity expression, which provides a space-to-time connection.

Justifications. Let $\left(\varphi_{\mathrm{o}}\right)$ and $\mathfrak{v}_{\mathrm{o}}$ be the reference values. Then the similarity ratios are $\varrho(\varphi)=\left(\varphi \mathrm{i} / \varphi_{\mathrm{o}}\right)$ and $\varrho(\mathfrak{v})=\left(\mathfrak{v} / \mathfrak{v}_{\mathrm{o}}\right)$. Therefore

$$
\varrho(\varphi)^{\mathrm{e}}+\varrho(\mathfrak{v})^{\mathrm{e}}=1 .
$$

Since $(\varphi \mathrm{i}) \mathrm{i}=\{$ distances $(\mathrm{L})$ and masses $(\mathfrak{m})\}$, one can write: $\mathfrak{m}_{\mathrm{o}} / \mathfrak{m}=\mathrm{L} / \mathrm{L}_{\mathrm{o}}$, so that

$$
\left(\mathfrak{m}_{\mathrm{o}} / \mathfrak{m}\right)^{\mathrm{e}}+\left(\mathfrak{v} / \mathfrak{v}_{\mathrm{o}}\right)^{\mathrm{e}}=1
$$

While coefficient (e) gets a value above unity, the geometry outside e $=1$ escapes the usual $(3-\mathrm{D}+\mathrm{t})$ space-time and, owing to the previously demonstrated necessity of an embedding 4-D (timeles) space, the coefficient (e) must reach e $=2$. Hence, the boundary conditions provide the following results:

$$
\begin{gathered}
\left(\mathfrak{m}_{\mathrm{o}} / \mathfrak{m}\right)^{2}+\left(\mathfrak{v} / \mathfrak{v}_{\mathrm{o}}\right)^{2}=1 \Leftrightarrow \mathfrak{m}=\mathfrak{m}_{\mathrm{o}} / \sqrt{1-\left(\mathfrak{v} / \mathfrak{v}_{\mathrm{o}}\right)^{2}} \\
\left(\mathrm{~L} / \mathrm{L}_{\mathrm{o}}\right)^{2}+\left(\mathfrak{v} / \mathfrak{v}_{\mathrm{o}}\right)^{2}=1 \Leftrightarrow \mathrm{L}=\mathrm{L}_{\mathrm{o}} \cdot \sqrt{1-\left(\mathfrak{v} / \mathfrak{v}_{\mathrm{o}}\right)^{2}}
\end{gathered}
$$


Table 2. The sequence of events occurring during a cycle of the behavior of the system composed of a moving particled deformation and its inertons cloud. Note: The velocity of transmission of the deformations from the particled ball to the degenerate balls is a nonlinear function of the following variables: $\mathrm{V}=$ $\mathrm{F}(\gamma, \mathrm{K}, \tau, \omega, \mathrm{R} ; f(\mathrm{e}))$ where $\gamma$ is the elasticity factor; $\mathrm{K}$ is the tenseness of the lattice; $\tau$ is the transmittivity of deformations; $\omega$ is the resistance of the lattice; $\mathrm{R}$ is the reaction to the emission of deformations; $f(\mathrm{e})$ is the fractal characteristic.

\begin{tabular}{|c|c|c|c|c|c|}
\hline $\begin{array}{l}\text { Phase } \\
\text { state }\end{array}$ & Start point & ] $0, \lambda / 2[$ & $\lambda / 2$ & ]$\lambda / 2, \lambda[$ & $\lambda$ \\
\hline $\begin{array}{l}\text { particled } \\
\text { ball }\end{array}$ & $\begin{array}{l}\max \text { velocity } v_{\mathrm{o}} \\
\max \text { mass } \mathrm{M}_{\mathrm{o}} \\
\max \text { tenseness; } \\
\max \text { fractal }(\mathrm{e})\end{array}$ & $\begin{array}{l}\text { mass loss; } \\
\text { velocity loss; } \\
\text { tenseness de- } \\
\text { crease }\end{array}$ & $\begin{array}{l}\text { min mass; } \\
\text { min velocity; } \\
\text { min tenseness; } \\
\text { balance with } \\
\text { cloud }\end{array}$ & $\begin{array}{l}\text { reincrease of } \\
\text { mass and ve- } \\
\text { locity }\end{array}$ & $\begin{array}{l}\text { return to } \\
\text { initial state } \\
\text { with max } \\
\text { mass and } \\
\text { velocity }\end{array}$ \\
\hline $\begin{array}{l}\text { inertons } \\
\text { cloud }\end{array}$ & $\begin{array}{l}\min \text { mass } \\
\mathrm{m}_{\mathrm{o}}=0 ; \\
\text { not yet resis- } \\
\text { tance to the mo- } \\
\text { tion of particled } \\
\text { deformation }\end{array}$ & $\begin{array}{l}\text { collisions with } \\
\text { lattice: emis- } \\
\text { sion of iner- } \\
\text { tons with high } \\
\text { speed (low lat- } \\
\text { tice tenseness) }\end{array}$ & $\begin{array}{l}\text { max mass; } \\
\text { max reaction; } \\
\text { max tenseness } \\
\text { as opposed to } \\
\text { particle motion; } \\
\text { max dispersion } \\
\text { of inertons by } \\
\text { the degenerate } \\
\text { lattice }\end{array}$ & $\begin{array}{l}\text { reaction decre- } \\
\text { ase; } \\
\text { return of mass; } \\
\text { decrease of } \\
\text { tenseness }\end{array}$ & $\begin{array}{l}\text { dissappea- } \\
\text { rance of } \\
\text { the inerton } \\
\text { cloud }\end{array}$ \\
\hline $\begin{array}{l}\text { whole } \\
\text { system }\end{array}$ & $\begin{array}{l}\text { no inerton re- } \\
\text { action; } \\
\text { resistance from } \\
\text { degenerate lat- } \\
\text { tice; }\end{array}$ & $\begin{array}{l}\text { tenseness ba- } \\
\text { lance and fle- } \\
\text { xibility of lat- } \\
\text { tice inertons } \\
\text { reaction }\end{array}$ & $\begin{array}{l}\text { balance in the } \\
\text { respective } \\
\text { tenseness and } \\
\text { center of mass }\end{array}$ & $\begin{array}{l}\text { reverse change } \\
\text { in localization } \\
\text { of the center } \\
\text { of mass }\end{array}$ & $\begin{array}{l}\text { de Broglie } \\
\text { period } \\
\text { reached }\end{array}$ \\
\hline
\end{tabular}

Remark 6.3. The Lagrangian $(\mathcal{L})$ should obey a similar law and $\left(\mathcal{L} / \mathcal{L}_{\mathrm{o}}\right)$ should fulfill relation $(6.4 \mathrm{~b})$ as a form of $\varrho(\varphi)^{\mathrm{e}}$. Then, $\left(\mathcal{L} / \mathcal{L}_{\mathrm{o}}\right)^{\mathrm{e}}+\left(\mathfrak{v} / \mathfrak{v}_{\mathrm{o}}\right)^{2}=1$ and analogically one could take $\mathcal{L}_{\mathrm{o}}=-\mathfrak{m} \mathfrak{v}_{\mathrm{o}}^{2}$. Thus finally $\mathcal{L}=-\mathfrak{m} \mathfrak{v}_{\mathrm{o}}^{2} \sqrt{1-\left(\mathfrak{v} / \mathfrak{v}_{\mathrm{o}}\right)^{2}}$.

By analogy with special relativity, $\mathcal{L}, \mathfrak{m}, \mathfrak{v}$ are the parameters of a moving object, while $\mathfrak{v}_{\mathrm{o}}=c$ where $c$ is the celerity of light. This supports some requirements pointed by Krasnoholovets (2001).

\subsection{Wave mechanics analysis}

Work (Krasnoholovets, 2002) has provided the derivation of wave equations from the inerton system. In short, given a set $\{\pi\}$ of two parameters describing the behavior of respectively the mass of the particle and the total inertons cloud (called the "rugosity" of the surrounding space, due to the distribution of scattered fragments of fractal deformations), the equations take the following general form:

$$
\ddot{\pi}-c_{\pi}^{2} \nabla \pi=0
$$


Solutions of (6.5) provide a real macroscopic wave function, allowing to find back the equivalent of the Schrödinger equation.

The same works finally derive also a mass field accounting for gravitation, and proves that the inert mass and the gravitational mass are the same. Hence, cosmic scale properties are inferring from particle scale characteristics.

In Part 3 of this work, further corollaries will be derived about the origin and classification of families of particled balls, and in connection with the former, it will be deduced an explanation on the mechanics underlying the origins of our observable space-time.

To which extent the motion of the $\{$ particle + inertons $\}$ system should follow the eddy-like motion that nonhomogeneous systems should follow (Lin and Wu, 1998) is matter of further investigations.

\section{Experimental assessment of the inertons existence}

Some preliminary experimental verifications have already been achieved, and some protocols for further proofs can be proposed.

\subsection{Former evidence}

The prediction of collective behaviour of atoms in solid matter from the existence of the $\{$ particle + inertons $\}$ system has been tested on both physical and chemical systems.

7.1.1. Moving electrons emit inerton clouds which can be detected in the form of anomalous photoelectric effects (Krasnoholovets, 2001b).

7.1.2. The impact of inertons on the collective behaviour of atoms in various metals has been evaluated and then experimentally observed by high resolution electron microscopy scanning (Krasnoholovets, and Byckov, 2000).

7.1.3. The existence of inerton clouds has been calculated for hydrogen atoms clustering in the $\delta$ - $\mathrm{KH}\left(\mathrm{IO}_{3}\right)_{2}$ crystal and the proton dynamic study has verified the theory (Krasnoholovets, 2001d).

\subsection{Further experimental protocols}

Former experiments have confronted the recording of signals from informational fields other than electromagnetic ones, like the Kozyrev effect and others (Kozyrev and Nazonov, 1978). Now, Krasnoholovets et al. (2001) have proposed a series of protocols for testing the emission of "inerton radiation" in various conditions. The measurements will be performed by using pyroelectric sensors constructed for this purpose. The project includes the following three cases:

7.2.1. Prediction of inerton emission by the sun, and observation of fluctuations of their field.

7.2.2. Prediction and measurement of the velocity of inerton waves emitted from distant stars whose parameters are sufficiently well known. 
7.2.3. Prediction and measurement of inerton flows emitted from satellites of solar planets.

\section{Discussion and conclusions}

The most important problem faced by most of physical theories is that they pose some assumptions which are founded on observations and speculations whose validity can hardly be supported excepted by indirect testing. About speculations, for instance classical theories pose the existence of elementary particles, string theory supposes the existence of free strings and of masses whose linear density gives the tension of the strings (Maldacena, 2000), while the rolling up of a membrane into a cylinder of infinitesimal diameter has been identified with a 1-D line (Duff, 1998), which is topologically wrong; lower boundaries are hypothesized for a Hamiltonian, if any, to account for a still ill-defined void state, while upper limits are required for the number of quantum states which are not postulated but resulting from statistical developments, and should be smoothed at large scales so as to let a discrete system be replaced by a continuous limit ('t Hooft, 1999). The latter point implicitly suggests that the observable universe is infinite, which remains an open question.

At observational levels, it remains difficult to get decisive answers for essentially two reasons. First, experimental data can often be interpreted in several ways: de Broglie noted that the same mathematical equations can have several [physical] explanations (Rothwarf, 1998), and Maldacena (2000) points that an inconsistent theory could agree with experiment. Second, even the measures of physical phenomena returns uncertain data: while the homogeneity and the isotropy of universe is required around each point for applicability of the FRW metrics (Smoller and Temple), the uniformity od universes appears as paradoxical (Bucher and Spergel, 1999) and the claim for anisotropy-supporting data (Ralston, Jain and Nodland, 1997) consistently raises polemic reactions (Ralston and Nodland, 1997).

Between these extreme positions, there unfortunately lies the situation in which no appropriate measure can decide between alternative hypothesis: this is the case for whether the expansion works for nearby galaxies only, or for the whole universe (Smoller and Temple, 2000), and what would be expanding between two independent objects (Walker, 1996; Bucher and Spergel, 1999). That objects can be independent, and why the expansion of "space" would not affect at all the microscopic world remain as many postulates, since the measuring devices may not be independent from the measured phenomena.

Our approach aimed at trying to pose as few postulates as possible, and to rather examine which kind of probationary space(s) and mathematical properties would fulfill the conditions required to support a proposition such as: there exists a physical universe embedding a self-perceived phenomenon that we use to call life. This drove to the identification of a primary axiom as the existence of the empty set, in turn providing existence of abstract mathematical spaces. Then, spaces of topologically closed objects gives raise to physical-like spaces, up to the function of self-conscious perception (Bounias, 2000). Several main aspects of this model will now be examined with respect to usual requirements for space-time structures and properties. 


\subsection{Space-time continuity and quantum structures}

Such a physical-like space is therefore composed of discrete cells endowed with quantumwise defined relative scales and whose interior is potentially provided with the power of continuum. An important consequence is that this property lays a bridge exactly on the gap separating the so-far discrete nature of microscopic world and the apparent continuity of the macroscopic universe.

The moments of junction map a timeless Poincaré section representing a state of the involved spaces into another state. Each Poincaré section may present some relationship with what t'Hooft (1999) called Cauchy surfaces of equal time. The moments of junction represent the interval between two successive states (each timeless) of a universe. Let $\mathrm{E}(\mathrm{i})$ be a Poincaré section like $\mathrm{S}(\mathrm{i})$ defined above: if it is an identity mapping, $\mathrm{MJ}=\operatorname{Id}(\mathrm{S})$, then there is no time interval from $\mathrm{S}(\mathrm{i})$ to $\mathrm{S}(\mathrm{i}+1)$. In all other cases, the MJ represents two important parameters: first, it accounts for a differential time interval, and then for a differential element of the geometry of the corresponding space. In this sense, it has neither "thickness" nor duration. There is no "distance" in the Hausdorff sense between $\mathrm{S}(\mathrm{i})$ and $\mathrm{S}(\mathrm{i}+1)$, just a change in the topological situation. Since the step from $\mathrm{S}(\mathrm{i})$ to $\mathrm{S}(\mathrm{i}+1)$ is a discrete one, it follows that: (i) the corresponding space owns discrete that is likely quantum properties and (ii) these discrete properties are valid whatever the scales, since they are founded on the set difference that is not dependent on any scale nor size of phenomena. It is noteworthy that these properties meet some requirement for space, time and matter, as suggested by t'Hooft (1999).

\subsection{Space-time and motion}

The moment of junction formalizes the topological characteristics of what is called motion, in a physical universe, that is what has been considered as needed for the understanding of physics (Rothwarf, 1998). While an identity mapping denotes an absence of motion, that is a null interval of time, a nonempty moment of junction stands for the minimal of any time interval: this meets a proposition of Sidharth (1999), stating that there should exist a minimum space-time interval and that "one cannot go to arbitrarily small space-time intervals or points". In our sense, there is no such "point": only instants which per se do not reflect timely features.

The need for morphisms of the topological structures as frame of reference as combined with the morphisms of objects interestingly meets with a requirement hypothesized by t'Hooft (1999): "beables" as commutable operators might be represented in morphisms of frames and the moments of junction, while "changeables" as non commutable components might be related to morphisms of objects, up to the not commutative biological components, which makes the transition to the next points.

Furthermore, morphisms are compatible with rotations that are to be expected in eddy-motion which stands at the basis of physical motion in a nonlinear universe (Wu and Lin, 2002). It should be noted that discontinuities may occur from changes occurring when a structure partly escapes the observable space-time through dimensional plunging into the embedding 4 -space and re-emerges in the perceived 3 -space. 


\subsection{Space-time reversibility properties}

It is noteworthy that the moment of junction is provided with reversibility, which accounts for the temporal reversibility of physical phenomena, as postulated by cosmologists (Smaller and Temple, 2000). In contrast, the biological arrow of time exhibits some irreversible features, since the sequence of brain mental images is founded on surjective mappings, which do not have the same fixed points in the reverse sense (Bounias, 2000b): this means that even if a biological system is physically reversible, the correspondence of mental images associated to outside perceptions with the the self of the perceiver would be changed.

\subsection{Topological constraints on space-time}

Seriu (2000) proposed a very interesting study on metric properties of a space of spaces. The author reaches the conclusion that physical constraints suggest the existence of drastic topological fluctuations at Planck scales. These observations would result in two correlative hypothesis: first, there would be sets of spaces with various topologies, and second, there would be scale-dependent topologies. Seriu acknowledges the risk that such considerations depart from the very foundations of topology as a mathematical concept. However, a mathematical space can give raise to several topologies, which range from coarser to finer forms, in an order relation (Bourbaki, 1990). In contrast with the smoothing at large scale recalled by t'Hooft (1999), here a smoothing of topologies at low scale would be needed. These apparent contradictions vanish with the properties of the empty hyperset providing discrete features at all scales, but also own the power of continuum, that is physical "continuity" inside each fundamental cell. Note that continuity in the mathematical sense does not require smoothing. Concerning the problem of scalerelated topological changes, it should be pointed that the set-distance is a scaleindependent measure, and would thus fulfill a requirement formerly raised in Part 1 of this study (Bounias and Krasnoholovets, 2003), in the form of an ultrafilter of the topologies required by Seriu. No contradiction seems to lurk in these approaches.

The spectra $\Delta^{\infty}$ proposed by Seriu should be invariant by spatial diffeomorphism: this implies continued differentiability, a property which is fulfilled by the convolution structure derived from our model. In addition, while the Laplacian used by Seriu as a probe for the geometry of the explored universe accounts for linear properties, the nonlinear convolution provides a generalization to nonlinear properties. While scale-dependent topologies may appear contradictory with fractal properties, our approach instead is consistent with such a structure

\subsection{Fractal space-time features}

Following the pioneer remarks of Mandelbrot (1989), some fractal properties have been found for the distribution of galaxies at rather small scales. However, the existence of a lower cut-off, as shared by the an spectra of Seriu (2000) seen above, precludes that the observed autocorrelations reflect a general fractality of the entire universe. In our model, the lattice that provides space and matter with their properties has been shown to meet the properties of a true fractal. This means that some fractal features should be shared by objects at all scales of our space-time. The contradiction of uniformity of Hubble law of isotropy of the background waves with the heterogeneity of a fractal geometry exhibiting voids and structures is just apparent. 
When Mandelbrot drew fractal galaxies on a sheet of paper, the "void" parts were represented by some lines connecting groups of points on the sheets. This would pose a problem only if an absolute void would be postulated between objects in the universe. In contrast, our approach suggests that a common fractal structure may hold on the whole of space as an embedding (nonmaterial) medium. It has been sketched here how this fractal geometry can account for the formation of matter corpuscles: due to all-scales self-similarity ratios, the same property should be extended to large scale formations. This would predict that similar fractal parameters should be found for both particle scales and cosmic scales. Lastly, that objects are constructed in and from a fractal lattice suggests that fractality if provided by the embedding medium: this answers a question raised by Cherbit (1987) and supports a former proposition of Feynman (1965) that the quantum trajectory of a particle is continued but not derivable.

\subsection{Predictions of fundamental physical parameters}

In the absence of "given" knowledge of what an object could be, Krasnoholovets and Ivanovsly (1993), Krasnoholovets (1997, 2000a,b, 2001a,b,c) and Krasnoholovets and Byckov (2000) proposed that a corpuscle could be represented by a local change in the geometry of a lattice. Independently, Bounias and Bonaly (1995, 1996, 1997) studied why a mathematical space could exist and how it could provide existence to a physical-like space. It came that the results support both the hypothesis of existence of a founding lattice of Krasnoholovets et al., and the prediction of emergence of the phenomenon of self-conscious perception, which could stand for a major characteristic of life (Bounias and Bonaly, 1997b, Bounias, 2000). Since the model makes useless the discrimination between relativistic and quantum approaches, we modestly expect that it might be fruitfully thought-provoking to the community, with emphasis on the fact that many various theories harbour correct elements that presently are diluted in a complex network of scattered hypothesis.

The next part of this study (Part 3) will provide a formal description of the construction and structures of the various fundamental particles allowed by the model and examine some implications up to cosmic scales and the origins and behavior of universes.

\section{References}

Blokhintsev, D., 1976. Principles of quantum mechanics, Russian, French edition 1981, MIR, Moscow, 683 pp.

Bolivar-Toledo, O., Candela Sola, S., Munoz Blanco, J.A., 1985. "Nonlinear data transforms in perceptual systems", Lecture Notes in Computer Sciences, Vol. 410, pp. 1-9.

Bonaly, A., 1992. Personal communication to M. Bounias.

Bonaly, A., Bounias, M., 1995. "The trace of time in Poincare sections of topological spaces", Physics Essays, Vol. 8, No. 2, pp. 236-44. 
Bounias, M., 1997. "Definition and some properties of set-differences, instances and their momentum, in the search for probationary spaces", Ultra Scientist of Physical Sciences, Vol. 9, No. 2, pp. 139-45.

Bounias, M., 2000a. "The theory of something: a theorem supporting the conditions for existence of a physical universe, from the empty set to the biological self", in Daniel M. Dubois, (Ed.) CASYS'99 Int. Math. Conf., Int. J. Computing Anticipatory Systems, Vol. 5, pp. 11-24.

Bounias, 2000b. "A theorem proving the irreversibility of the biological arrow of time, based on fixed points in the brain as a compact or delta-complete space", Am. Inst. Phys. Conf. Proc., Vol. CP517, pp. 233-43.

Bounias, M., 2001. "Indecidability and Incompleteness In Formal Axiomatics as Questioned by Anticipatory Processes", in Daniel M. Dubois (Ed.) CASYS'2000 Int. Math. Conf., Int. J. Computing Anticipatory Systems (in press).

Bounias, M., Bonaly, A., 1996. "On metrics and scaling : physical coordinates in topological spaces", Indian Journal of Theoretical Physics, Vol. 44, No. 4, pp. 303-21.

Bounias, M., Bonaly, A., 1997a. "The topology of perceptive functions as a corollary of the theorem of existence in closed spaces", BioSystems, Vol. 42, pp. 191-205.

Bounias, M., Bonaly, A., 1997b. "Some theorems on the empty set as necessary and sufficient for the primary topological axioms of physical existence", Physics Essays, Vol. 10, No. 4, pp. 633-43.

Bounias, M., Krasnoholovets, V., 2003. "Scanning the structure of ill-known spaces: Part 1. Founding principles about mathematical constitution of space", Kybernetes: The Int. J. of Systems \& Cybernetics, Vol. 32, No. 7/8, pp. 945-75 (also physics/0211096).

Bourbaki, N., 1990a. Theorie des ensembles, Masson, Paris, Chapters 1-4, p. 352.

Bourbaki, N., 1990b. Topologie Gendrale, Masson, Paris, Chapters 1-4, p. 376.

Boyer, T., 2000. "The infinitely empty does not exist". Pour La Science (Scientific American, French edition), Vol. 278, pp. 128-37.

Bucher, M., Spergel, D., 1999. "L'inflation de 1' univers", Pour La Science (Scientific American, French edition), Vol. 257, pp. 50-7.

Cherbit, 1987. "Dimension locale, quantite de mouvement et trajectoires", in Fractals, Masson, Paris, 340-52.

Duff, M., 1998. "The new string theory", Pour La Science (Scientific American, 
French edition), Vol. 246, p. 68.

Einstein, A., 1920. Aether and the theory of relativity. Leyden University Lecture. French translation: Gauthier-Villars, Paris, pp. 1-12 (1992).

Feynman, R., Hibbs, A., 1965. Quantum mechanics and path integrals, McGraw Hill, NY, p. 176.

Keilman, Y., 1998. "On the breakdown of the principle of relativity", Physics Essays, Vol. 11, No. 2, pp. 325-29.

Kozyrev, N.A., Nasonov, V.V., 1978. In Astronometry and celestian mechanics, Akademia Nauk SSSR, Moscow, Leningrad, pp. 168-79 (in Russian).

Krasnoholovets, V., Ivanovsky, D., 1993. "Motion of a particle and the vacuum", Physics Essays, Vol. 6, No. 4, 554-63. (Also arXiv.org e-print archive quant-ph/9910023).

Krasnoholovets, V., 1997. "Motion of a relativistic particle and the vacuum", Physics Essays, Vol. 10, No. 3, pp. 407-16. (Also quant-ph/9903077).

Krasnoholovets, V., Byckov, V., 2000. "Real inertons against hypothetical gravitons. Experimental proof of the existence of inertons", Indian Journal of Theoretical Physics, Vol. 48, No. 1, pp. 1-23. (Also quant-ph/0007027).

Krasnoholovets, V., 2000. "On the nature of spin, inertia and gravity of a moving canonical particle", Indian Journal of Theoretical Physics, Vol. 48, No. 2, pp. 97-132. (Also quant-ph/0103110).

Krasnoholovets, V., 2001a. "On the theory of anomalous photoelectric effect stemming from a substructure of matter waves", Indian Journal of Theoretical Physics, Vol. 49, No. 1, pp. 1-32. (Also quant-ph/9906091).

Krasnoholovets, V., 2001b. "Space structure and quantum mechanics", Space-time \& Substance, Vol. 1, No. 4, pp. 172-75. (Also quant-ph/0106106).

Krasnoholovets, V., 2001c. "On the way to submicroscopic description of nature", Indian Journal of Theoretical Physics, Vol. 49, No. 2, 81-95. (Also quant-ph/9908042).

Krasnoholovets, V., 2001d. "Collective dynamics of hydrogen atoms in the $\mathrm{KIO}_{3} \cdot \mathrm{HIO}_{3}$ crystal dictated by a substructure of the hydrogen atoms' matter waves", cond-mat/0108417

Krasnoholovets, V., Strokach, O., Skliarenko, Akimov, L. 2001. Inerton astronomy: Facility for measuring the inerton radiation from stars and planets, project. 
Lin, Y., Wu, Y., 1998. "Blown-ups and the concept of whole evolutions in systems science", Problems of Nonlinear Analysis in Engineering Systems, Vol. 4, pp. 16-31.

Maldacena, J., 2000. "Gravity, particle physics and their unification", eConf C990809, pp. 840-52. (Also arXiv.org e-print archive hep-th/0002092).

Marinov, S., 1996. "Cosmological aspects of the absolute space-time theory", Physics Essays, Vol. 9, No. 3, pp. 357-67.

Okun, L.B., 1988. Physics of elementary particles, Nauka, Moscow (in Russian), p. 92.

Ralston, J., Jain, P., Nodland, B., 1997. "The corscrew effect", Phys. Rev. Lett., Vol. 816, pp. 26-9.

Ralston, J.P., Nodland, B., 1997. "An update on cosmological anisotropy in electromagnetic propagation", in Donnelly T.W. (Ed.) Proc. 7th Internat. Conf. on the Intersection of Particle and Nuclear Physics, Big Sky, Montana, Amer. Inst. Phys. CP .(Also astro-ph/978114.)

Sidharth, B.C., 1999. "The fractal universe: from the Planck scale to the Hubble scale", quant-ph/9907024.

Seriu, M., 2000. "Space of spaces as a metric space", Commun. Math. Phys., Vol. 209, pp. 393-405.

Smoller, J., Temple, B., 2000. "Cosmology with a shock-wave", Commun. Math. Phys., Vol. 210, pp. 275-308.

t'Hooft, G., 1999. "Quantum gravity as a dissipative deterministic system", Class. Quantum Grav., Vol. 16, pp. 3263-279.

Walker, F.L., 1996. "The expanding space paradox: can the galaxies really recede?", Physics Essays, Vol. 9, No. 2, pp. 209-15.

Wu, Y., Lin, Y., (2002). "Beyond nonstructural quantitative analysis", in Blown ups, spinning currents and modern science, World Scientific, New Jersey, London, p. 324 .

\section{Further reading}

Bounias, M., Bonaly, A., 1994. "On mathematical links between physical existence, observability and information: towards a "theorem of something", Ultra Scientist of Physical Sciences, Vol. 6, No. 2, pp. 251-9.

Hales, T.C., 2000. "Cannonballs and honeycombs", Notices of the AMS, Vol. 47, 
No. 4, pp. 440-9.

Hannon, R.J., 1998. "An alternative explanation of the cosmological redshift", Physics Essays, Vol. 11, No. 4, pp. 576-8.

Havard, G., Zinsmeister, M., 2000. "Thermodynamic formalism and variations of the Hausdorff dimension of quadratic Julia sets", Commun. Math. Phys., Vol. 210, pp. $225-47$.

Joyce, M., Anderson, P.W., Montuori, M., Pietronero, L., Sylos Labini, F., 2000. "Fractal cosmology in an open universe", Europhysics Letters, Vol. 49, pp. 416-22.

Lavrentiev, M.M., Eganova, I.A., Lutset, M.K., Fominykh, S.F., 1990. "About distance influence of stars on resistor", Proc. Acad. Sci. USSR, Vol. 314, No. 2, pp. 352-5 (in Russian). 